\title{
Shock Waves and Commutation Speed of Memristors
}

\author{
Shao Tang, ${ }^{1}$ Federico Tesler, ${ }^{2}$ Fernando Gomez Marlasca, ${ }^{3}$ Pablo Levy, ${ }^{3}$ \\ V. Dobrosavljević, ${ }^{1}$ and Marcelo Rozenberg ${ }^{4}$ \\ ${ }^{1}$ Department of Physics and National High Magnetic Field Laboratory, \\ Florida State University, Tallahassee, Florida 32306, USA \\ ${ }^{2}$ Departamento de Física-IFIBA, FCEN, Universidad de Buenos Aires, \\ Ciudad Universitaria Pabellón I, (1428) Buenos Aires, Argentina \\ ${ }^{3}$ GIA-CAC-CNEA, Avenida Gral Paz 1499 (1650) San Martín, Pcia Buenos Aires, Argentina \\ ${ }^{4}$ Laboratoire de Physique des Solides, CNRS, Université Paris-Sud, \\ Université Paris-Saclay, 91405 Orsay Cedex, France
}

(Received 18 November 2014; revised manuscript received 20 October 2015; published 15 March 2016)

Progress of silicon-based technology is nearing its physical limit, as the minimum feature size of components is reaching a mere $10 \mathrm{~nm}$. The resistive switching behavior of transition metal oxides and the associated memristor device is emerging as a competitive technology for next-generation electronics. Significant progress has already been made in the past decade, and devices are beginning to hit the market; however, this progress has mainly been the result of empirical trial and error. Hence, gaining theoretical insight is of the essence. In the present work, we report the striking result of a connection between the resistive switching and shock-wave formation, a classic topic of nonlinear dynamics. We argue that the profile of oxygen vacancies that migrate during the commutation forms a shock wave that propagates through a highly resistive region of the device. We validate the scenario by means of model simulations and experiments in a manganese-oxide-based memristor device, and we extend our theory to the case of binary oxides. The shock-wave scenario brings unprecedented physical insight and enables us to rationalize the process of oxygen-vacancy-driven resistive change with direct implications for a key technological aspect-the commutation speed.

DOI: 10.1103/PhysRevX.6.011028

The information age we live in is made possible by a physical underlayer of electronic hardware, which originates in condensed-matter physics research. Despite the great progress made in recent decades, the demand for faster and power-efficient devices continues to grow. Thus, there is urgent need to identify novel materials and physical mechanisms for future electronic-device applications. In this context, transition metal oxides (TMOs) are attracting much attention for nonvolatile memory applications [1]. In particular, TMO are associated with the phenomenon of resistive switching (RS) [2] and the memristor device [3], which is emerging as a competitive technology for nextgeneration electronics [1,4-10]. The RS effect is a large, rapid, nonvolatile, reversible change of the resistance, which may be used to encode logic information. In the simplest case, one may associate high and low resistance values to binary states, but multibit memory cells are also possible $[11,12]$.

Published by the American Physical Society under the terms of the Creative Commons Attribution 3.0 License. Further distribution of this work must maintain attribution to the author(s) and the published article's title, journal citation, and DOI.
Subject Areas: Condensed Matter Physics,

Materials Science, Nonlinear Dynamics
Typical systems where RS is observed are two-terminal capacitor-like devices, where the dielectric might be a TMO and the electrodes are ordinary metals. The phenomenon occurs in a strikingly large variety of systemsranging from simple binary compounds, such as $\mathrm{NiO}$, $\mathrm{TiO}_{2}, \mathrm{ZnO}, \mathrm{Ta}_{2} \mathrm{O}_{5}, \mathrm{HfO}_{2}$, and $\mathrm{CuO}$, to more complex perovskite structures, such as superconducting cuprates and colossal magnetoresistive manganites $[2,4,6,9,13]$.

From a conceptual point of view, the main challenges for a nonvolatile memory are as follows: (i) to change its resistance within nano seconds (required for modern electronics applications), (ii) to be able to retain the state for years (i.e., nonvolatile), and (iii) to reliably commute the state hundreds of thousands of times.

Through extensive experimental work in the past decade, a consensus has emerged around the notion that the change in resistance is due to migration of ionic species, including oxygen vacancies $\left(V_{\mathrm{O}}\right)$, across different regions of the device, affecting the local transport properties of the oxide [14]. In particular, the important role of highly resistive interfaces, such as Schottky barriers (SBs), has also been pointed out $[7,9,15]$.

In contrast with the experimental efforts, theoretical studies remain relatively scarce [16-24]. A few 
phenomenological models were proposed and numerically investigated, which captured different aspects of the observed effects [3,25-27].

In this context, gaining theoretical insight is of the essence. Thus, in the present work, we address one of the key aspects of the RS phenomenon, namely, the issue of the commutation speed of the resistance change. Our first striking result is a connection between the RS phenomenon and shock-wave formation, a classic topic of nonlinear dynamics [28]. In fact, we argue that the profile of oxygen vacancies that migrate during the resistive change forms a shock wave that propagates through the Schottky barrier and leaks onto the bulk of the device, which we schematically illustrate in Fig. 1. We further validate the scenario by means of numerical simulations on a successful model of RS and by novel experiments done on a manganese-based memristor device. Both model calculations and experiments reveal a striking scaling behavior as predicted by the shock-wave scenario.

Generalized Burgers' equation. When ions migrate through a conducting medium under the influence of strong applied voltage, they are likely to undergo a nonlinear diffusion process, as we explain in the following. The total ionic current $\mathbf{j}(t, \mathbf{x})=\mathbf{j}_{\text {diffusion }}+\mathbf{j}_{\text {drift }}$ can be expressed as the sum of a diffusion current $\mathbf{j}_{\text {diffusion }}=-D \nabla u$ and a drift current $\mathbf{j}_{\text {drift }}$, which is induced by the local electric field $\mathbf{E}$ and the local concentration $u$. Together with the continuity equation $\partial_{t} u+\nabla \cdot \mathbf{j}(t, \mathbf{x})=0$, this immediately gives us a generalized diffusion equation of the Nernst-Planck type. This would represent a familiar drift-diffusion equation if the local electric field $\mathbf{E}$ was held constant, i.e., independent of the local ion concentration $u$. In contrast, in (poorly) conducting media and under voltage pulses, the local electric field may strongly depend on the local ion concentration; this effect is the key source of nonlinearity causing the formation of shock waves and very sudden resistance switching $[7,8]$.

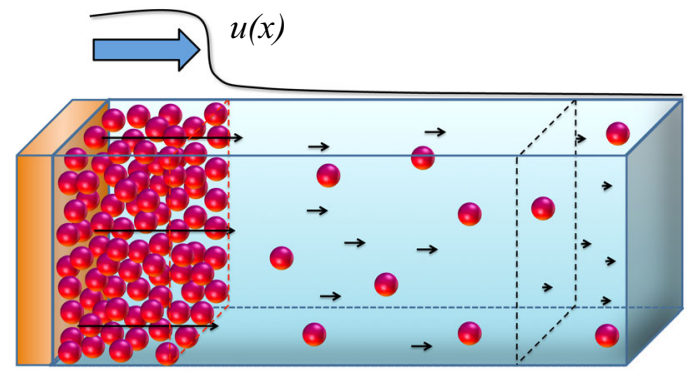

FIG. 1. Schematic representation of the shock-wave evolution. The orange region indicates the metallic electrode, and the blue indicates the TMO dielectric. Small spheres denote the ionic defects (oxygen vacancies) whose density profiles form a shock wave. It evolves through a highly resistive (Schottky) interface and eventually leaks over the more conductive bulk, producing the resistive change. Black arrows depict the strength of the local electric fields.
Since electrons move much faster than the ions, we can view the ions as static when considering the electronic current $\mathbf{I}$, which obeys a steady-state condition $\nabla \cdot \mathbf{I}=0$. The local electric field is then simply determined, through Ohm's law, by the local resistivity $\mathbf{E}=\rho(u) \mathbf{I}$, which may be a strong function of the local ion concentration $u$. In particular, in bad metals such as the transition metal oxides, the migrating ions (e.g., oxygen vacancies) act as scattering centers for the conduction electrons. In such situations, we expect $\rho(u)$ to be a monotonically increasing function of the local ion vacancy density $u(t, \mathbf{x})$. Therefore, the redistribution of the local ion density results in the change of local resistivities and, consequently, of the local electric fields, which further promotes the nonlinear effect in the drift.

Under the experimentally relevant case where the transverse currents may be neglected, the problem simplifies to a one-dimensional nonlinear diffusion equation,

$$
\partial_{t} u+f(u) \partial_{x} u=D \partial_{x x} u
$$

where $f(u) \equiv \partial_{u} j_{\text {drift }}(u, I)$, and $I(t)$ is the magnitude of the electronic current. Equation (1) can be considered as a generalization of the famous Burgers' equation, which corresponds to the special case $f(u) \propto u$. Its most significant feature is the presence of a density-dependent drift term, which physically means that the "crest of the wave" experiences a stronger external force than the "trough." This generally leads to the formation of a sharply defined shock-wave front in the $u(t, x)$ profile, which assumes a universal form at long times, completely independent of the-quickly "forgotten"-initial conditions. Although the process is driven by the drift term, the stability of the shockwave form is provided by the existence of the diffusion term which prevents the shock wave from self-breaking [28-30]. Remarkably, the formation of shock waves proves to be robust in a much more general family of models with the nonlinear drift term specified by the function $f(u)$, any monotonically increasing function of $u$. The qualitative behavior can be established by using the well-known method of characteristics [28,31], which we illustrate with an example in Fig. 2 (see Appendix A for a more detailed explanation).

The drift current is generally given by the expression $j_{\text {drift }}=u g(E)$. The form of the function $g(E)$ is material dependent, and here we envision two limiting situations. In homogeneous conductors, we should have simple "Ohmic" behavior as $g(E) \sim E$, while in granular materials, we expect exponential dependence due to activated transport, corresponding to $g(E) \sim \sinh \left(E / E_{0}\right)$, where $E_{0}$ is a parameter describing the activation process.

Remarkably, these general ideas find an explicit realization in the context of RS in transition metal oxide memristors, such as manganites [15,32]. In fact, their transport properties are very sensitively dependent on the oxygen stoichiometry, i.e., on the concentration of oxygen 


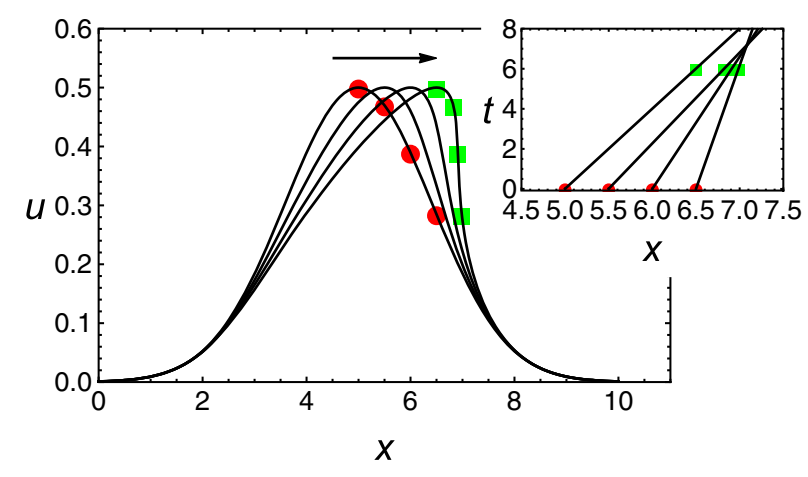

FIG. 2. Time evolution of a generic density profile according to the generalized Burgers' equation (1). Curves correspond to profiles at $t=0,2,4$, and 6 . The (arbitrary) initial profile is taken to be Gaussian, and we adopt $f(u) \propto u^{2}$. The color dots track constant- $u$ points and show the formation of the universal steep shock-wave profile. Inset: $t-x$ coordinates of the constant- $u$ tracking points (characteristics). The velocity of each point only depends on its $u$ value. The crossing of the characteristics signals the formation of the multivalued steep shock-wave front.

vacancies $\left[V_{\mathrm{O}}\right]$. Thus, it is now widely accepted that the mechanism of the bipolar (i.e., polarity-dependent) RS in those systems is due to the induced changes in the spatial distribution of $\left[V_{\mathrm{O}}(\mathbf{x})\right] \equiv u(\mathbf{x})$ by means of externally applied strong electric stress [7,8]. In particular, the accumulation of vacancies within highly resistive regions between the oxide and the metallic electrode, such as SB interfaces, greatly increases the (two-terminal) resistance across the device [27]. This accumulation can be achieved by applying strong voltage pulses across the device, leading to the high-resistance state $R_{\mathrm{HI}}$. Abrupt resistance switching from such a high-resistance state to a significantly lower-resistance state can be accomplished by reversing the voltage applied, which removes a significant fraction of vacancies from the SB region. The precise characterization of this resistance-switching process is the main subject of this paper.

We should mention that an important assumption is that the nonlinear drift term plays the dominant role compared to the normal diffusion; i.e., we shall not be concerned with the resistive changes involving thermal effects $[7,8]$. This restriction enables us to apply our analytical tools in a simple manner, allowing us to obtain a simplified mathematical description of the migration process, as we show in the following.

Model system. For concreteness, we adopt the voltage-enhanced oxygen-vacancy migration model [27] (VEOVM), which corresponds to granular materials with an activated transport process, and it has previously been used for manganite devices [27]. Within the framework of this model, we perform numerical simulations to validate our shock-wave scenario. The VEOVM simply assumes that the local resistance of the cell at (discretized) position $x$ along the conductive path of the device is simply given as a linear function of the local vacancy concentration, namely,

$$
r(x)=A_{\alpha} u(x),
$$

with $\alpha=\mathrm{SB}, \mathrm{B}$, where SB denotes the highly resistive (Schottky-barrier) region and B the more conductive bulk [15]. The values of these constants are taken as $A_{\mathrm{SB}} \gg A_{\mathrm{B}}=1$, which allows us to neglect the bulk resistance [27]. The discretized conducting path assumes the metal electrode at $x=0$, and $x=x_{\text {int }}$ denotes the point within the dielectric where the SB meets the bulk region. Under the action of the external stress (electric current $I$ ), the local fields at each cell position $x$ are computed at every discrete time step $t$. The field-driven migration of vacancies is simulated by computing the local ionic migration rates from cell $x$ to $x+\Delta x$ as [27]

$$
\begin{aligned}
& P(x, x+\Delta x) \\
& \quad=u(x)[1-u(x+\Delta x)] \exp \left(\frac{-V_{0}+q \operatorname{Ir}(x)}{k_{B} T}\right),
\end{aligned}
$$

where, for simplicity, we take the ionic charge $q=1$ and $k_{B} T=1$. The parameter $V_{0}$ denotes the activation energy for ionic diffusion. The new profile $u(t, x)$ is updated from the migration rates, and from Eq. (2) we get the new total (two-point) $R(t)$ as the discrete $x$ integral of the local cell's resistance $r(t, x)$. Here, for simplicity, we focus on a single active SB-bulk interface, while the more general situation with two barriers may be analyzed following a similar line of argument [27]. The applied external electric stress that we adopt is a constant current, in both simulations and experiments (see below).

As described in Ref. [27], the initial vacancy concentration profile is assumed to be constant $[u(x)]=\left[u^{0}\right]$. The "forming" or initialization of the memory is done by first applying a few current loops of alternate polarity, $\pm I^{0}$, until the migration of vacancies evolves towards a limit cycle, with a well-defined profile $u_{0}(x)$. After this, the system begins to repetitively switch between two values: $R_{\mathrm{HI}}$ and $R_{\mathrm{LO}}$. In the first value, most of the vacancies reside within the high-resistance region $\mathrm{SB}$, and in the second, vacancies accumulate in the more conductive bulk. The $R_{\mathrm{HI}}$ state with the vacancies piled up in the first cell, at $x=1$, defines the initial state for the shock-wave propagation (see Fig. 3).

Shock-wave formation: The "propagation phase". We apply an external field with polarity pointing from the SB to the bulk and observe the evolution of the vacancy profile as a function of the (simulation) time. As can be observed in Fig. 3, there is a rapid evolution of the profile into a shockwave form with a sharply defined front. We also notice that the total resistance remains approximately constant during an initial phase and suddenly starts to decrease after the front hits the internal SB-bulk interface at $x_{\text {int }}$ (inset of top panel of Fig. 3). We analyze these key features in the following.

First, we focus on the propagation of the shock-wave front position $x_{s}(t)$, as shown in Fig. 3 (bottom-left 

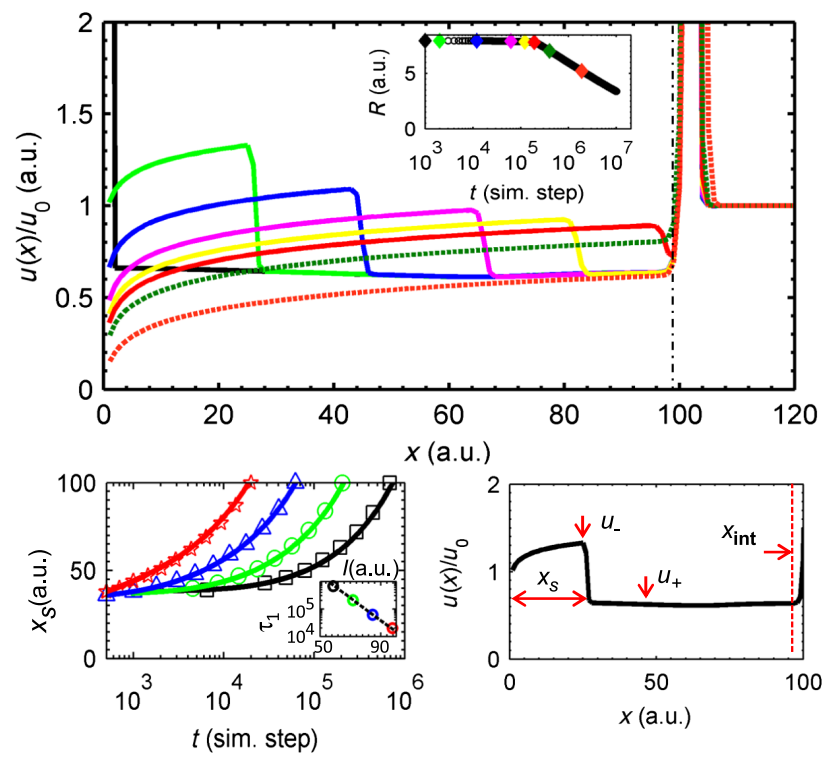

FIG. 3. Top panel: Snap shots of the time evolution of the density profile $u(t, x)$ within the active interfacial region in a simulation of the VEOVM model (see Appendixes C and D for details). The current used is $I[$ a.u. $]=71.5$, with $A_{\mathrm{SB}}=1000$ and $A_{\mathrm{B}}=1$. where a.u. stands for arbitrary units in all corresponding figures. The time steps of the successive profiles can be read off from the corresponding color dots in the inset. The initial state $u(0, x)$ (black line) exhibits a vacancy pileup next to the electrode at $x=1$. The SB-bulk interface is denoted with a vertical dashdot line at $x_{\text {int }}=100$. The large accumulation of vacancies on the right of $x_{\text {int }}$ (bulk side) results from the initial "forming" cycles performed on an originally uniform profile of density $u_{0}$. The result of the forming cycles is an approximately fixed background on top of which the density profile further evolves (see details in Ref. [27]). Inset: Resistance of the device as a function of time. Color dots indicate the value of $R(t)$ at the corresponding snapshots of the main panel. Bottom-left panel: Evolution of the shock-wave front position $x_{s}(t)$ for different currents $(I[\mathrm{a} . \mathrm{u}]=58.5,71.5,84.5$, and 97.5). Dots are from numerical simulations, and the solid lines are analytic fits from integration of Eq. (4). Inset: Characteristic impact time $\tau_{1}$ as a function of applied currents from the numerical simulation (circles) and analytic fit (dotted line) in semilog scale. Bottom-right panel: Shock-wave parameters.

panel) for different values of the electronic current $I$. We observe that the characteristic time $\tau_{1}$ for the shock wave to travel through the Schottky barrier and reach the SB-bulk point $x_{\text {int }}$ decreases exponentially with the magnitude of $I$. To obtain analytical insight for this behavior, we recall that the velocity of the shock-wave front $d x_{s} / d t$ is very generally given by the RankineHugoniot conditions [28,33], which express it as the ratio of the spatial discontinuity of the (vacancy) drift current and the spatial discontinuity of the density profile across the shock viz. $d x_{s} / d t=\Delta j /\left.\Delta u\right|_{x_{s}}$. Within the VEOVM model [27], we obtain the following nonlinear rate equation (see Appendix B for details), which describes the dynamics of the shock-wave front: $\frac{d x_{s}}{d t}=\frac{2 D u_{-} \sinh \left(I A_{\mathrm{SB}} u_{-}\right)-2 D u_{+} \sinh \left(I A_{\mathrm{SB}} u_{+}\right)}{\Delta u}$,

where $D$ is a prefactor related to the activation energy for vacancy migration (Arrhenius factor) [see Eq. (1) and Ref. [27]), and $u_{-/+}$are the densities of vacancies at the two sides of the shock-wave front (see Fig. 3). The density $u_{-}$depends on the shock-wave front position via $u_{-}=Q / x_{s}+u_{+}$, where $Q$ is the total number of vacancies carried by the shock wave, which remains a constant parameter through the propagation phase $\left(t<\tau_{1}\right)$ and $u_{+}$is a constant background density which can be written as $u_{+}=Q_{B} / x_{\text {int }}$, with $Q_{B}$ the total number of background vacancies.

Our description of the propagation phase is fully consistent with our numerical simulations. As shown in the inset of Fig. 3 (top panel), the resistance remains essentially constant until the wave front reaches the SB-bulk interface after a (current-dependent) time $\tau_{1}$, and then begins to drop. Moreover, we also achieved a good fit to the shock-front velocity by using Eq. (4), as is shown in Fig. 3 (see Appendix E for details).

Resistance switching: The "leakage phase". After the shock front reaches the boundary point $x_{\text {int }}$, the resistance begins to drop. To understand this behavior, we note that the total resistance of the Schottky barrier is given by the total number of vacancies within the barrier region viz. from Eq. (2), $R_{\mathrm{SB}}=\int_{\mathrm{SB}} d x A_{\mathrm{SB}} u(x)$. As a result, the resistance drop per unit time is approximately given by the ionic vacancy current passing through the SB-bulk interface at $x_{\text {int }}$,

$$
d R(t) / d t=-A_{\mathrm{SB}} j\left(x=x_{\mathrm{int}}\right),
$$

since $R_{\mathrm{SB}} \gg R_{\mathrm{B}}$ as $A_{\mathrm{SB}} \gg A_{\mathrm{B}}$. Notice that during the propagation phase, the ionic current through the interface $x_{\text {int }}$ is negligibly small. This is because the initial vacancy concentration there, and hence the local field, is also negligibly small. However, when the shock-wave front eventually reaches the end of the SB region, after traveling for a time $\tau_{1}$, we do expect a sudden resistance drop as a large number of ionic vacancies begin to leak out into the bulk region.

We now focus on the detailed description of the resistive drop. In Fig. 4, we show the systematic dependence of $R(t)$ as a function of the applied external (electronic) current. Along with the simulations of the VEOVM, we also present our experimental results measured on a manganite-based $\left(\mathrm{La}_{0.325} \mathrm{Pr}_{0.300} \mathrm{Ca}_{0.375} \mathrm{MnO}_{3}\right)$ memristive device [34-38]. The experimental setup and device are described in detail in Appendix F and in Ref. [39]. The set of curves were obtained for applied current intensities just above the threshold for the onset of the resistance switch. The goal here is not to demonstrate the fast switching speed of the device but rather to achieve relatively slow switching speeds in order to access the different time scales. In 

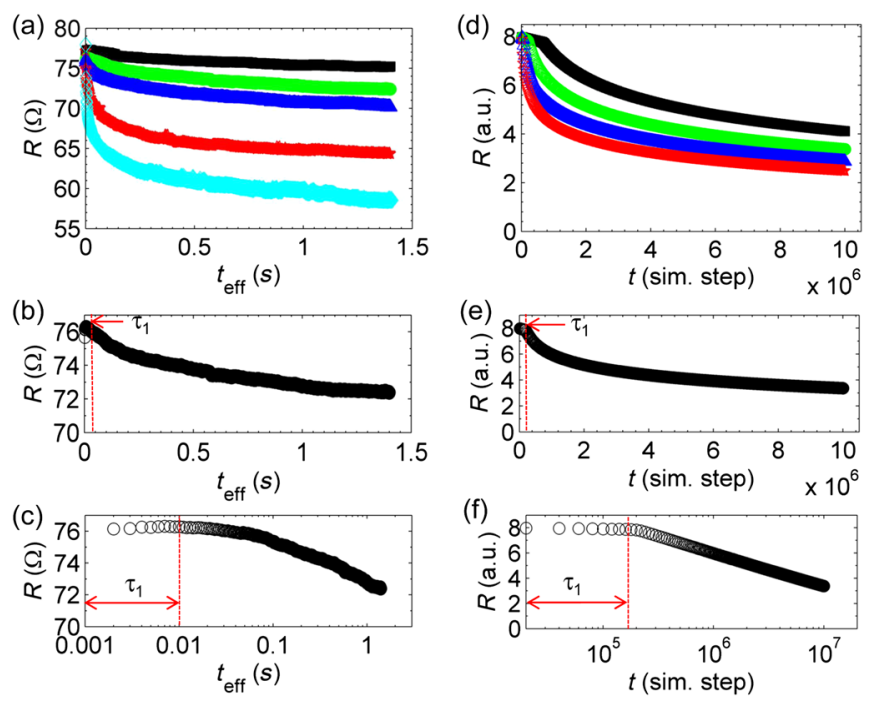

FIG. 4. Time dependence of the resistive change $R(t)$ for various external current intensities. Top left: Experimental data measured on an Ag/LPCMO/Ag memristor with $I=37.5 \mathrm{~mA}$ (black), $40 \mathrm{~mA}$ (green), $50 \mathrm{~mA}$ (blue), $80 \mathrm{~mA}$ (red), and $100 \mathrm{~mA}$ (cyan). Here, $t_{\text {eff }}$ is the effective time duration of the applied currents (see Appendix F for details). The initial state was reset by applying an intense negative polarity current of $350 \mathrm{~mA}$. Note that the initial value of the resistance, $78 \Omega$, is recovered within $\pm 1 \Omega$. Top right: Model simulations with applied current: $I[$ a.u. $=58.5$ (black), 71.5 (green), 84.5 (blue), and 97.5 (red). Middle panels: Experimental data for $I=40 \mathrm{~mA}$ (left) and simulations for $I[$ a.u. $]=71.5$ (right). Here, $\tau_{1}$ is defined as the time interval from the beginning of the applied pulse until the resistance starts to drop. Bottom panels: Idem as middle panels in a semilog scale.

addition, this also minimizes thermal heating effects [39]. We observe that in both simulation and experiments, the resistance change rapidly becomes larger and faster with the increase of the applied electric stress intensity. We also observe an overall good qualitative agreement between experiments and model simulations. This is also highlighted by the semilog plots, which clearly display the twostage process involved in the resistive switch, before and after the impact time $\tau_{1}$.

Remarkably, within a shock-wave scenario, we may also obtain explicit expressions that quantify the resistance change during the leakage phase. Our analysis may be simplified by first noting, from general considerations of shock waves, that their shape at long times becomes "flat"; i.e., the gradient of the local density rapidly decreases $\left(\partial_{x} u \rightarrow 0\right)$ at all points that were overtaken by the shockwave front $[28,31]$. Indeed, our data are fully consistent with this observation, as the vacancy density profile within the SB remains approximately flat [i.e., spatially constant, $\left.u(t, x)=u_{\mathrm{SB}}(t)\right]$ at all times after the shock front reaches the interface (see Fig. 3). Then, within the VEOVM, the SB resistance is simply proportional to the total vacancy concentration within the barrier, and we have
$R(t) \approx R_{\mathrm{SB}}(t)=A_{\mathrm{SB}} x_{\mathrm{int}} u_{\mathrm{SB}}(t)$. Since the electronic current $I$ is held fixed, the vacancy (i.e., ionic) current through the interface depends only on the vacancy concentration $u_{\mathrm{SB}}$ [cf. Eq. (3)]. Thus, within the VEOVM, we obtain a nonlinear rate equation, describing the resistance drop during the "leakage phase":

$$
\frac{d R}{d t}=-\frac{2 D R}{x_{\mathrm{int}}} \sinh \left(\frac{I R}{x_{\mathrm{int}}}\right)
$$

Similar to what was shown before for the propagation phase, this equation may be validated by a quantitative fit to the simulation results (see Appendix E). Note that because of the strong nonlinear form of this rate equation, the $R(t)$ response is significantly different from the simple exponential decay expected in the familiar linear case (e.g., in standard RC circuits). Therefore, within the short time scale associated with the initial fast drop of resistance and where the RS is significant $\left(I R / x_{\text {int }} \gg 1\right)$, the present type of nonlinear system is dominated by the activated process and the approximation $\sinh \left(I R / x_{\text {int }}\right) \approx \frac{1}{2} \exp \left(I R / x_{\text {int }}\right)$ is valid. This enables the approximate solution of Eq. (6),

$$
R=R_{\mathrm{HI}}-\frac{x_{\mathrm{int}}}{I} \ln \left(1+\frac{t^{*}}{\tau_{2}(I)}\right)
$$

where the time $t^{*}$ is measured from the "impact" time $\tau_{1}$ and $\tau_{2}(I)=\left(x_{\text {int }}^{2} / D I R_{\mathrm{HI}}\right) \exp \left(-I R_{\mathrm{HI}} / x_{\text {int }}\right)$ (see Appendix G) is the current-dependent characteristic time for the resistance drop.

Resistivity scaling. An interesting consequence of Eq. (7) is that it suggests the scaling behavior of the curves $R\left(t^{*}\right)$. In fact, one may define the normalized resistance drop $\delta R\left(t^{*}\right)=\left[R-R\left(\tau_{2}\right)\right] /\left[R_{\mathrm{HI}}-R\left(\tau_{2}\right)\right]$ and see from Eq. (7) that it obeys the scaling form

$$
\delta R\left(t^{*}\right)=1-\ln \left(1+t^{*} / \tau_{2}\right) / \ln (2) .
$$

In Fig. 5, we demonstrate that this striking feature is indeed present in both our experiments and simulations data. In the upper panels of the figure, we show the excellent scaling that is achieved, where all the experimental and simulation curves $R(t)$ from Fig. 4 were respectively collapsed into a single curve. Moreover, the collapsed data can also be fitted with a slightly more general form of Eq. (8), which we discuss in Appendix G. Remarkably, in the lower panels of Fig. 5, we show that a collapse of the data $R(t)$ can also be obtained using the impact time $\tau_{1}$ as the scaling variable. This is significant because it shows that a single scaling behavior may include the two phases of the resistive switching process, namely, before and after $\tau_{1}$. We should mention that the scaling scenario was derived with the assumption of an Ohmic behavior in the $I-V$ characteristics. While this may not be the case in general [39], within the present set of 

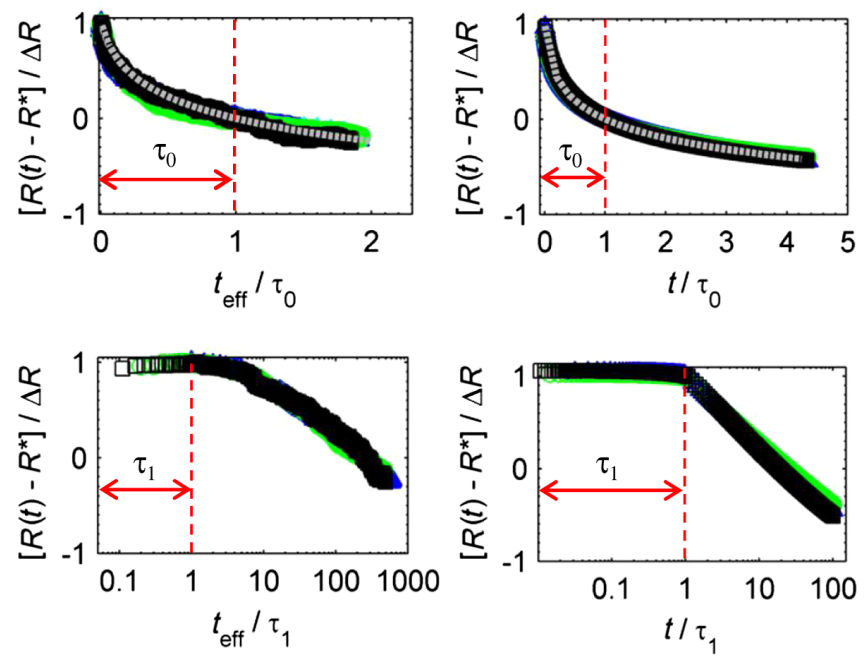

FIG. 5. Scaled curves of the $R(t)$ data sets of Fig. 4. The left panels show the collapsed experimental data and the right ones the numerical simulations. The time $\tau_{0}$ is an auxiliary scaling variable, which is proportional to the characteristic time $\tau_{2}$ (see Appendix $\mathrm{G}$ for details on the scaling procedure). The scaled data were fitted (white dotted line) with a generalized version of Eq. (8) (see Appendix G). The lower panels show the same data sets scaled with the shock-wave impact time $\tau_{1}$. (The experimental curves show only three data sets for the lower current values. At higher currents, our electronics could not resolve $\tau_{1}$.) To achieve the scaling of the lower panels, we assumed for each plot the normalization value of $\Delta R$ determined from the previous scaling (top panels).

experiments, which are performed near the current threshold of RS, our results indicate that this is a reasonable assumption or at least a valid approximation.

To conclude, from quite general considerations of migration of ionic defects under strong electric fields in solids, we have argued that the dynamics of the spatial profile of defect concentration should be governed by a Burgers'-type nonlinear equation and develop shock waves. We demonstrated that this scenario is indeed realized within a concrete realization, namely, a ionic migration model that was previously applied to describe resistive switching phenomena in manganite-based memristive devices. In those systems, a key role is played by the migration of oxygen vacancies, which are the ionic defects relevant to the electronic transport properties. We thus predicted a two-stage process for the resistive switch phenomenon. In the initial stage, the oxygen-vacancy concentration profile develops a shock wave that propagates throughout a highly resistive (Schottky-barrier) region near the electrode. During this phase, the resistance essentially does not change. This is followed by a second phase, where the shock wave emerges from the highresistive region and the ionic defects leak into the conductive bulk. Our scenario was further validated by novel experimental data on a manganite-based memristor device. A remarkable result of our study is that both the numerical simulations and the experimental curves obeyed a scaling behavior, providing decisive support to our theory. The present work provides novel insights on the physical mechanism behind the commutation speed on novel nonvolatile electronic memories, unveiling an unexpected connection between a phenomenon of technological relevance and a classic theme of nonlinear dynamical systems. Furthermore, and from a practical point of view, our study unveils some of the key physical parameters that control the resistive switching properties of devices. Of foremost importance are the characteristic time scales $\tau_{1}$ and $\tau_{2}$. Interestingly, we find that both depend on the same combination of parameters, namely, $x_{\text {int }}^{2} /\left(D I R_{\mathrm{HI}}\right)$ and $x_{\text {int }} /\left(I R_{\mathrm{HI}}\right)$, which appear in the exponential factor [see Eq. (7), and Eqs. (G3) and (G8) in Appendix G]. While we may intuitively understand that a shorter interface or a stronger current pulse would increase the commutation speed, this expression also provides concrete guidance for the engineering effort of devices, which is less evident. For instance, it shows that shortening the length of the interface by a factor of 3 should at least gain a full order of magnitude in speed. It also points towards adopting materials where the diffusion constant of oxygen vacancies is large. While this is a priori fixed by the specific chemistry of the material, one may also envision that the diffusion might be boosted by preparing samples with smaller grain sizes and with extended columnar defects that could be induced by heavy-ion irradiation.

A final important point to consider regards the general validity of the present results for other types of resistive switching systems, for instance, those based on $\mathrm{HfO}_{x}$, $\mathrm{TaO}_{x}$, and $\mathrm{TiO}_{2-x}$, which are currently receiving a great deal of attention. Those compounds, in their stoichiometric form, are, unlike PCMO, very good insulators. Thus, in order to make RS devices, one possibility is to perform an initial "electroforming" procedure, which creates metallic filaments that may subsequently be "burned" and regenerated, irrespective of the applied polarity of the electric pulses. That mechanism is not relevant for the present study. However, there is another possibility to make RS devices with these compounds, which is to heavily dope very thin films (about tens of $\mathrm{nm}$ ) with oxygen vacancies [40]. In this case, the systems become more conductive than their respective stoichiometric compound and display a qualitatively different type of resistive switching under electric pulsing, which is bipolar and relevant here. In fact, in these systems, the mechanism relays on oxygen-ionic migration under the strong electric fields; thus, it is interesting to note that the shock-wave formation may be at work, shaping the evolution of the spatial profile of oxygen density. Indeed, a local increase of oxygen concentration brings these systems (locally) closer to their respective stoichiometric composition; thus, it locally increases the resistivity. Hence, we realize that, for such systems, one may fulfill the conditions for the onset of 
shock-wave propagation. In fact, an analogous role to that of oxygen-vacancy density in PCMO (that renders the system more resistive) may be played in the simpler binary compounds by the oxygen density (see Appendix I for details and the simulation results of Ref. [22]). It would be very interesting indeed to explore the fast commuting behavior in thin films of $\mathrm{TaO}_{x}, \mathrm{HfO}_{x}$ and related compounds, to search for evidence of shock-wave propagation. These are exciting directions that are now open for future investigation.

\section{ACKNOWLEDGMENTS}

This work was partially supported by public grants from the French National Research Agency (ANR), Project LACUNES No. ANR-13-BS04-0006-01 (M. R.), the NSF Grants No. DMR-1005751 and No. DMR-1410132 (S. T. and V. D.), the University of Buenos Aires (UBACyT 2013-2016), and the MinCyT PICT2013-0788 Project MeMOSat (F. T., F. G. M., P. L., and M. R.).

\section{APPENDIX A: METHOD OF CHARACTERISTICS}

In this section, we demonstrate that shock waves are typically formed in the presence of sufficient nonlinearity, and we analyze the problem via the method of characteristics. Considering the vacancy migration in highly resistive barriers, where we can neglect the normal diffusion term $D \partial_{x x} u$, we obtain a first-order, partial differential equation of the general form

$$
\partial_{t} u+c(u, t) \partial_{x} u=0 .
$$

Here, the $u$ dependence in $c(u, t)$ gives rise to the nonlinearity effect for the onset of shock-wave formation. In order to determine the density profile evolution, we need to track the motion of points corresponding to specific values of $u$ (see Fig. 2, left panel). We can solve the trajectories (the so-called "characteristics") followed by such a point in the $t-x$ plane by solving the following equation $[28,31,41]$ :

$$
\frac{d x}{d t}=c(u, t) .
$$

As different characteristics might intersect with each other in the $t-x$ plane (see the inset of Fig. 2), the emergence of intersecting trajectories indicates that the solution $u(t, x)$ becomes multivalued since the points can be traced back along each of the characteristics to different initial values of $u(t=0, x)$. The intersection that happens chronologically first determines the formation of the shock wave, as shown in Fig. 2. As an illustration, we provide an example with $c(u, t)=u^{2}$ and an initial Gaussian distribution $\quad u(t=0, x)=0.5 \exp \left[-(x-5)^{2} / 4\right]$. The time-dependent wave profile is solved numerically with fixed boundary conditions, and its characteristics are computed according to Eq. (A2).

We should stress, however, that this qualitative behavior is a robust feature of any such nonlinear diffusion equation, provided that the prefactor $c(u, t)$ is a monotonically increasing function of $u$ [i.e., $\partial_{u} c(u, t)>0$ ]. To see this, note that Eq. (A1) takes the form of a simple wave equation; thus, its solution is a traveling wave, with speed locally proportional to $c(u, t)$. Therefore, at any given time, each point on the wave front moves with a different speed proportional to $c(u, t)$, and the "crest" having the largest value of $u$ moves fastest, which leads to a "kink" type shock. Alternatively, it is easy to check that if $\partial_{u} c(u, t) \leq 0$, the shock wave will not form. In the case where $c(u, t)$ is any function independent of $u$, the characteristics are curves of the same shape but parallel to each other; hence, they would not have any intersections; again, the shock wave would not form. Finally, it can be shown that if the diffusion term $D \partial_{x x} u$ is nonzero but parametrically small (as compared to the drift term), the diffusion will prevent the wave from overtopping (undergoing "self-breaking"), and the shock-wave front will remain sharp as it propagates $[28,31]$.

\section{APPENDIX B: DYNAMICS OF SHOCK WAVES}

In this section, we provide a simple derivation of the Rankine-Hugoniot condition [28,33], which determines the equation of motion of the shock-wave front. Important features of the shock wave are the spatial discontinuities of $u$ and $j$. Considering a shock-wave front propagating on the interval $[0, d]$ in one dimension, we define $u_{+} \equiv u\left(t, x_{s}(t)+\varepsilon\right), \quad u_{-} \equiv u\left(t, x_{s}(t)-\varepsilon\right)$, $j_{+} \equiv j\left(t, x_{s}(t)+\varepsilon\right)$, and $j_{-} \equiv j\left(t, x_{s}(t)-\varepsilon\right)$, where $x_{s}(t)$ is the coordinate of the shock-wave front and $\varepsilon \rightarrow 0^{+}$is a infinitesimal positive quantity. According to the continuity equation $\partial_{t} u+\partial_{x} j=0$, we can write

$$
\begin{aligned}
& \frac{d}{d t}\left(\int_{0}^{x_{s}(t)} u d x+\int_{x_{s}(t)}^{d} u d x\right)=j(0)-j(d), \\
& \frac{d x_{s}}{d t} u_{-}-\frac{d x_{s}}{d t} u_{+}+\int_{0}^{x_{s}(t)} \partial_{t} u d x+\int_{x_{s}(t)}^{d} \partial_{t} u d x \\
&=j(0)-j(d) .
\end{aligned}
$$

Using the continuity equation again, we can immediately determine the shock-wave front velocity via the following equation:

$$
v_{s}=\frac{d x_{s}}{d t}=\frac{j_{+}-j_{-}}{u_{+}-u_{-}}=\left.\frac{\Delta j}{\Delta u}\right|_{x_{s}},
$$

which is often called the Rankine-Hugoniot condition. 


\section{APPENDIX C: VEOVM MODEL}

As was mentioned in the main text, to simulate the vacancy dynamics, we adopted the voltage-enhanced oxygen-vacancy migration model (VEOVM), which is a wellvalidated model for the RS effect [13,27]. Taking a capacitor-like device, this model considers a onedimensional conductive channel connecting the two contacts, along which oxygen vacancies can migrate through (see Fig. 6). This channel is divided into small cells corresponding to physical nano-domains, and at the same time, the whole device is divided into two regions: the active interfacial region close to the metal contact (i.e., high-resistive Schottky barrier) and the high-conductive bulk region. A diagram of the model with a single active contact, as used in the simulations, is presented in the top panel of Fig. 6. The resistance of each cell in the channel $r(x)$ is proportional to the local density of vacancies: $r(x)=A_{\alpha} u(x)$, where $u(x)$ is the density of vacancies on the cell located at $x$ and $A_{\alpha}$ is a proportionality constant whose magnitude depends on the region of the device; here, $\alpha=\mathrm{SB}, \mathrm{B}$, where SB stands for the Schottky barrier and B for the bulk, and where $A_{\mathrm{SB}} \gg A_{\mathrm{B}}$. Migration is assumed to occur only between neighbor cells, and in each step of the simulation, the probability for vacancy migration from a cell at $x$ to its neighbor at $x+\Delta x$ is computed according to

$P(x, x+\Delta x)=u(x)(1-u(x+\Delta x)) \exp \left(\Delta V(x)-V_{0}\right)$,

where $\Delta V(x)$ is the drop of voltage (per unit length) at $x$ and $V_{0}$ is an activation constant for vacancy migration. In each step of the simulation, the migration from cell to cell is computed, and the new resistance of each cell is calculated. The total resistance of the device is calculated simply as the addition of all the cells in the channel. As the typical value of $u(x)$ in simulation varies between $10^{-4}$ and $10^{-3}$, the middle term $(1-u(x+\Delta x))$ can and will be neglected in the theoretical analysis explained in the following sections. As mentioned in the main text, this model corresponds to the case of granular materials with activated transport process. To put it in the context of the generalizedBurgers' equation description made in the main text, it is easy to see that the drift current originating from this model satisfies the general form $j_{\text {drift }} \sim \sinh (E)$. The net current of vacancies generated by the action of an external
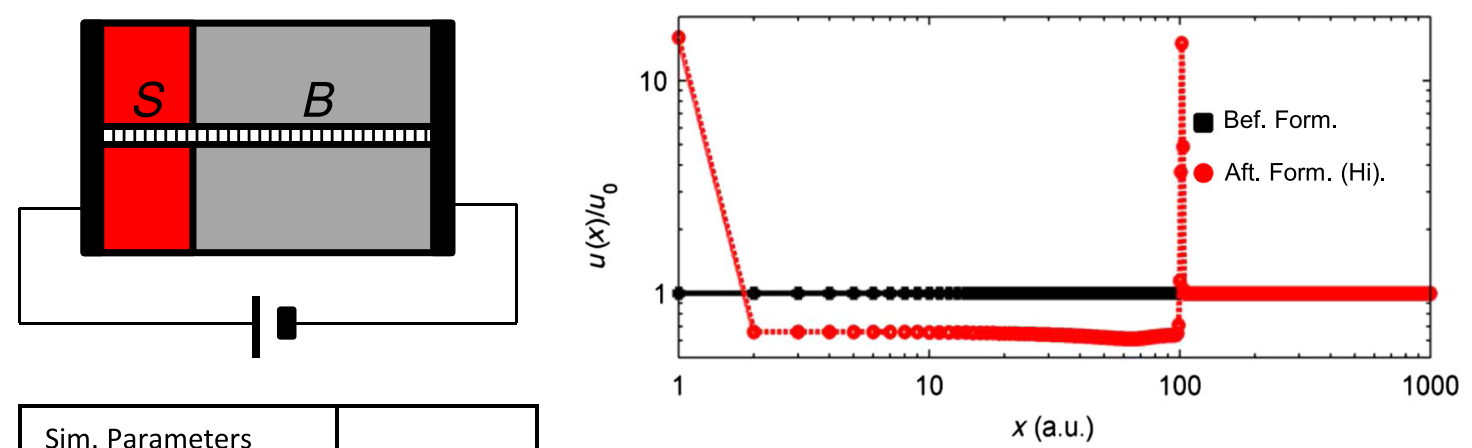

\begin{tabular}{|l|l|}
\hline Sim. Parameters & \\
\hline$u_{0}$ (vacancies/cell) & $1 \times 10^{-4}$ \\
\hline$A_{S B}$ (a.u.) & 1000 \\
\hline$A_{B}$ (a.u.) & 1 \\
\hline$V_{0}$ (units of kT) & 16 \\
\hline$L$ (total \# of cells) & 1000 \\
\hline$X_{\text {int }}$ (int. \# of cells) & 100 \\
\hline
\end{tabular}

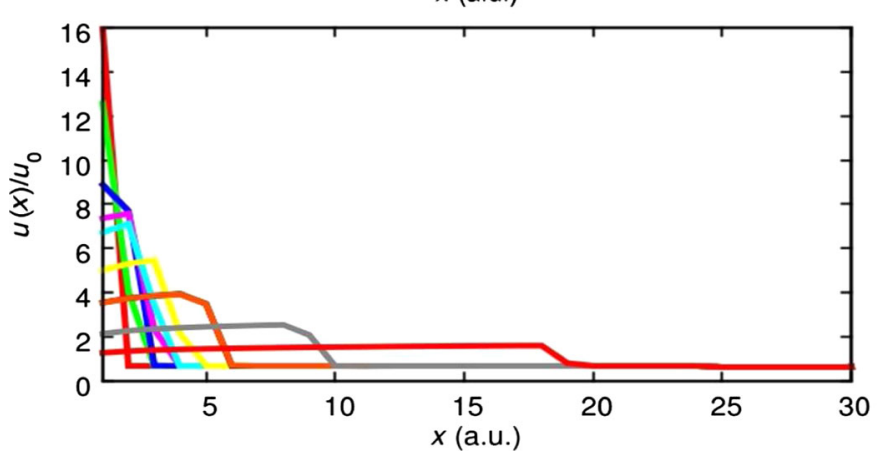

FIG. 6. Top-left panel: Schematic diagram of the VEOVM model with a single active contact. The two regions SB and B correspond to the high-resistance interface (Schottky barrier) and the more conductive central bulk, respectively. The small cells within the channel indicate the domains. Top-right panel: Vacancy distribution along the conductive channel before (black squares) and after (red circles) the forming process. The distribution shown after the forming process corresponds to a HI resistance state. Starting from a uniform distribution, during the forming process a characteristic distribution is obtained where vacancies initially at the interface migrate to the low-field bulk region, generating a pileup of vacancies close to the limit between the two regions. In the HI state, some of these vacancies migrate back to the interfacial region and are accumulated in the vicinity of the metal contact. Bottom left (table): parameters used in the simulations. Bottom-right panel: Snapshots of the shock-wave formation in the early stage of simulations (between the first and second snapshots of Fig. 3). 
electric field $E(x)=\partial V(x) / \partial x$, between a cell at $x$ and its neighbor cell at $x+\Delta x$, can be written simply as Fick's law for the migration probability: $j_{\text {drift }}=\partial P / \partial x \approx$ $[P(x, x+\Delta x)-P(x+\Delta x, x)] / \Delta x$, where in the last term the discrete character of the model has been introduced. In particular, for our model, we take $\Delta x=1$. Using the definition of $P$ from the model (neglecting its middle term), we get $j_{\text {drift }}=P(x, x+\Delta x)-P(x+\Delta x, x)=$ $2 D u \sinh (\Delta V(x))$, where $D$ stands for the Arrhenius factor $\exp \left(-V_{0}\right)$. If the external electric stress is a controlled current $I$, then $\Delta V(x)=\operatorname{Ir}(x)=I A_{\alpha} u(x)$.

\section{APPENDIX D: SIMULATION DETAILS}

In order to analyze the existence of a shock-wave scenario, the HI to LO process was simulated for different external currents. Previously, an initial forming process was performed at which current of different polarities were applied and well-defined HI and LO states were obtained. In the top-right panel of Fig. 6, the vacancy profile is shown before and after the forming process. The device starts with a uniform distribution along the channel, and by the end of the forming process, the profile shows a characteristic distribution where the vacancies migrated out from the interfacial region and accumulated in the neighboring lowfield bulk region [27]. The figure shows the formed HI resistance state, where we can see a second accumulation of vacancies next to the left metal contact in the interfacial region. This second accumulation of vacancies constitutes the initial distribution for the $\mathrm{HI}$ to LO process, and it will evolve to form the shock wave during switching, as shown in the main text. To improve the stability of the simulations, the currents were applied using a rise time (from 0 to their actual values) that is always negligible compared with the characteristic times of the process, $\tau_{1}$ and $\tau_{2}$ (with rise times on the order of a few thousand steps). The simulated device contained a total of 1000 cells, with a 100-cell-long interfacial region (left interface). For the initial distribution, a uniform density of $u_{0}=1 \times 10^{-4}$ per cell was used. The value of the activation constant $V_{0}$ was set to 16 , which corresponds to the activation energy of $0.4 \mathrm{eV}$ reported in similar manganite systems [42] (i.e., $0.4 \mathrm{eV} / k_{B} T=$ $0.4 / 0.026$ at room temperature). For the resistivity constants, we used $A_{\mathrm{SB}}=1000$ and $A_{\mathrm{B}}=1$ (for a table with the parameters, see Fig. 6). For the analysis developed in this paper, we solely consider the shock wave after it is formed. In the bottom panel of Fig. 6, we show a standard formation process of the shock wave: the initial narrow accumulation against the metal contact rapidly evolves into the shock-wave profile.

\section{APPENDIX E: FITTING FOR THE VEOVM MODEL}

In this section, we explain the details of the comparison between simulation and theory and the procedures used for fitting. Consider the drift current within the left interfacial region in the VEOVM model:

$$
\begin{aligned}
j(u, t, x) & =P(x, x+\Delta x)-P(x+\Delta x, x) \\
& =2 D u \sinh \left(I A_{\mathrm{SB}} u\right),
\end{aligned}
$$

where the term $1-u(x+\Delta x)$ in the probability has been neglected as explained before. From the Rankine-Hugoniot condition derived in Eq. (B3), an expression for the shockwave front velocity $d x_{s} / d t$ can be obtained, as provided in Eq. (4) in the main text. Performing a redefinition of parameters (for practical reasons only), we can rewrite such equation as

$$
\frac{d x}{d t}=\frac{2 D}{x_{\mathrm{int}}}\left[\left(1+\frac{\alpha}{\beta} x\right) \sinh \left(\frac{I}{\beta}+\frac{I}{\alpha x}\right)-\frac{\alpha}{\beta} x \sinh \left(\frac{I}{\beta}\right)\right],
$$

where $u_{-}=u_{+}+\Delta u, Q=\Delta u x_{s}$ is the total number of vacancies carried by the shock wave, $Q_{B} \equiv u_{+} x_{\text {int }}$ is the total number of background vacancies in the left region (with $x_{\text {int }}$ the length of the interfacial region), $x \equiv x_{s} / x_{\text {int }}$ is the normalized coordinate, and we defined the new parameters $\alpha \equiv x_{\text {int }} / A_{\mathrm{SB}} Q$ and $\beta \equiv x_{\text {int }} / A_{\mathrm{SB}} Q_{B}$. From the previous definitions, we can write the high-resistance value as $R_{\mathrm{HI}}=A_{\mathrm{SB}}\left(Q_{B}+Q\right)$, where $R_{\mathrm{HI}}$ is a constant determined by the vacancy concentration and independent of $I$ [43].

We can then solve this equation numerically with the material-dependent parameters $x_{\text {int }}, \alpha, \beta$, and $D$. Considering the initial rise time for the current and the nonflatness of the shock wave, an accurate test of the $x_{s}(t)$ prediction can be performed for $x>x_{0} \approx 0.4$ using the integral-form equation

$t-t_{0}=\int_{x_{0}}^{x} \frac{\left(x_{\text {int }} / D\right) d y}{2\left(1+\frac{\alpha}{\beta} y\right) \sinh \left(\frac{I}{\beta}+\frac{I}{\alpha y}\right)-\frac{2 \alpha}{\beta} y \sinh \left(\frac{I}{\beta}\right)}$,

which is used for the fit of the simulation data for $t<\tau_{1}$ in the lower-left panel of Fig. 3 in the main text.

On the other hand, we also have the rate equation for the switching (i.e., "leakage") phase [cf. Eq. (6) in the main text]. The numerical solution of this equation is used to fit the simulation data in the resistance-switching phase for $t>\tau_{1}$ as shown in Fig. 7.

The values of the parameters that enter Eqs. (E3) and (6) were extracted directly from the simulation results. We find $Q_{B}=63.4 u_{0}, Q=15.3 u_{0}, A_{\mathrm{SB}}=1000, R_{\mathrm{HI}}=7.9$ a.u., and $D=1.12 \times 10^{-7}$. The very good fits of the simulation data shown in Figs. 3 and 7 were achieved by slightly relaxing the value of the single parameter $x_{\text {int }}=100$ to the 


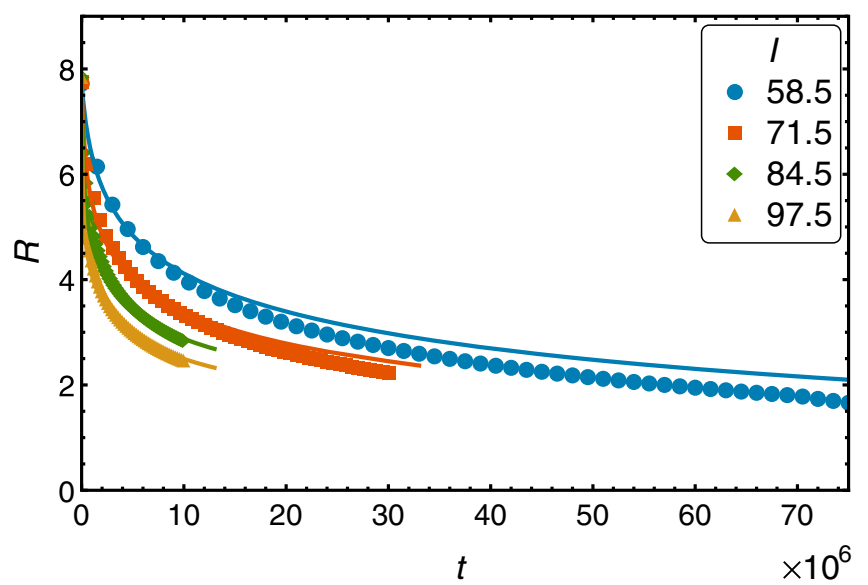

FIG. 7. Evolution of the resistance during the switching phase for different applied currents according to simulations (dots) and theory (lines) from Eq. (6). The currents shown are $I=58.5$ a.u., 71.5 a.u., 84.5 a.u. and 97.5 a.u.

values 94.3 (Fig. 3) in the propagation phase and 82.2 (Fig. 7) in the leakage phase.

The small discrepancy between the relaxed parameters and the actual value mainly comes from the nonflatness of the density profile both during the propagation of the shock wave and during the leaking phase, as well as from the fact that a small part of background vacancies leaks into the bulk during the propagating phase.

\section{APPENDIX F: EXPERIMENTAL DETAILS}

For the experimental validation of our theory, the RS phenomenon was studied in a bulk $\mathrm{La}_{0.325} \mathrm{Pr}_{0.300} \mathrm{Ca}_{0.375} \mathrm{MnO}_{3}$ (LPCMO) polycrystalline sample with hand-painted $\mathrm{Ag}$ contacts of approximately $1-\mathrm{mm}$ diameter. The device is a parallelepiped of $1 \times 1 \times 10 \mathrm{~mm}^{3}$, with the contacts painted over the $10-\mathrm{mm}$ surface. The distance between contacts is also on the order of $1 \mathrm{~mm}$. Measurements were performed using a three-wire configuration in order to develop a single contact analysis.

To induce the RS effect, an external constant current was used as the electric stimulus. To generate this current and to acquire the data, a Keithley 2612 Sourcemeter was employed. The measurements were done using a threewire configuration in order to measure a single-interface resistance [Fig. 8(a)]. The HI to LO RS was studied for currents of different magnitude, as described in the main text. More information about the measurement technique and simultaneous contact analysis can be seen in Ref. [34].

The application of the current is done following a pulsed protocol: A high-current pulse (Write) is followed by a low-current pulse (Read). A schematic diagram of the pulsed protocol is shown in Fig. 8(b). Each pulse lasts $1 \mathrm{~ms}$. Between two pulses, there is an interval of

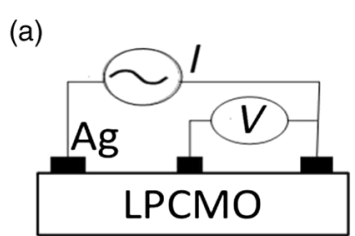

(c)
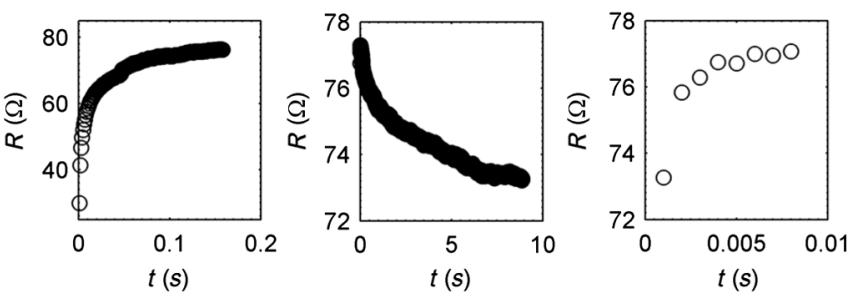

FIG. 8. (a) Diagram of the experimental setup. (b) Sketch of the pulsed current protocol used in the experiments. A highcurrent Write pulse (blue) is followed by a low-current Read pulse (red). The first pulse generates the RS, while the second pulse measures the nonvolatile resistance. (c) $R$ vs $t$ curves from a complete RS process. The system is first taken to a HI resistance state under a current of $-350 \mathrm{~mA}$ (left panel). Then, the $\mathrm{HI}$ to LO RS is measured under an applied current of $37.5 \mathrm{~mA}$ (middle panel). Finally, the system is taken back, i.e., "reinitialized," to a HI resistance state with the application of a $-350 \mathrm{~mA}$ current.

about $0.5 \mathrm{~s}$, meant to reduce possible heating effects. The time axis exhibited in the $R$ vs $t$ curves [both in Fig. 4 and in Fig. 8(c) below] is the effective time elapsed during the actual application of the Write pulses, i.e., disregarding both the 0.5 -s timeout and the Reading elapsed time. The Write pulses possess enough strength to change the resistive state of the system, while the Read pulse (low current) measures the remnant (stable, nonvolatile) resistance of the device without affecting it. In the experiments shown in the main text, the Write pulses are on the order of the $\mathrm{mA}$, while the Read pulses are on the order of the $\mu \mathrm{A}$.

The complete measurement process is shown in Fig. 8(c). To obtain the initial HI state, pulses of $-350 \mathrm{~mA}$ were applied [Fig. 8(c), left panel]. Next, the desired accumulation experiment is performed by applying positive pulses of constant amplitude which decrease the resistance [Fig. 8(c), middle panel]. The initial HI-resistance state is recovered by applying $-350-\mathrm{mA}$ pulses [Fig. 8(c), right panel]. In every case, the initial HI-resistance state obtained was of the same magnitude within a range of about $1 \%$.

\section{APPENDIX G: SCALING}

In this section, we show that the scaling behavior is a direct consequence of the strong nonlinearity of the drift current $j(u, t, x)$, which depends exponentially on the local electric field. In the time range where signifi- 
cant resistive switching occurs, we have $\left(I R / x_{\text {int }} \gg 1\right)$, and we may approximate $\sinh \left(I R / x_{\text {int }}\right) \approx \frac{1}{2} \exp \left(I R / x_{\text {int }}\right)$. From Eq. (6), using a normalized resistance $\tilde{R}=R / R_{\mathrm{HI}}$, we have

$$
\frac{d \tilde{R}}{d t}=-\frac{D}{x_{\mathrm{int}}} \exp \left[\left(\frac{I R_{\mathrm{HI}} \tilde{R}}{x_{\mathrm{int}}}\right)(1+\lambda)\right]
$$

where $\lambda=\left(x_{\mathrm{int}} / R_{\mathrm{HI}}\right) \ln \tilde{R} /(I \tilde{R})$ is a small parameter as long as $\tilde{R}$ is close to 1 (i.e., at the beginning of the resistive change). For simplicity, we consider the solution at the leading order by setting $\lambda=0$ :

$$
\tilde{R}=1-\frac{x_{\mathrm{int}}}{I R_{\mathrm{HI}}} \ln \left(1+\frac{t^{*}}{\tau_{2}}\right),
$$

where $t^{*}=t-\tau_{1}$ is the time measured from the impact time, as explained in the main text, and $\tau_{2}$ is a characteristic time for the resistance switch, dominated by an exponential dependence of the applied current as follows:

$$
\tau_{2}=\frac{x_{\mathrm{int}}^{2}}{D I R_{\mathrm{HI}}} \exp \left(-\frac{I R_{\mathrm{HI}}}{x_{\mathrm{int}}}\right)
$$

In Fig. 9, we use this approximate expression to fit the $R(t)$ simulation data. Comparison with the previous fit done with Eq. (6) and shown in Fig. 7 allows us to check that this approximate solution is relatively accurate within the time domain we are interested in for fast switching devices.

Now, using Eq. (G2), we can show that the $I$-dependent family curves $R(t)$ should obey scaling. We first consider the normalized resistive change $\delta R(t)$ defined as

$$
\delta R(t)=\frac{R(t)-R\left(\tau_{0}\right)}{R_{\mathrm{HI}}-R\left(\tau_{0}\right)}
$$

where $\tau_{0}$ is some yet-unspecified time (and we drop the * from $\left.t^{*}\right)$. Then, replacing $R(t)$ with Eq. (G2), we have

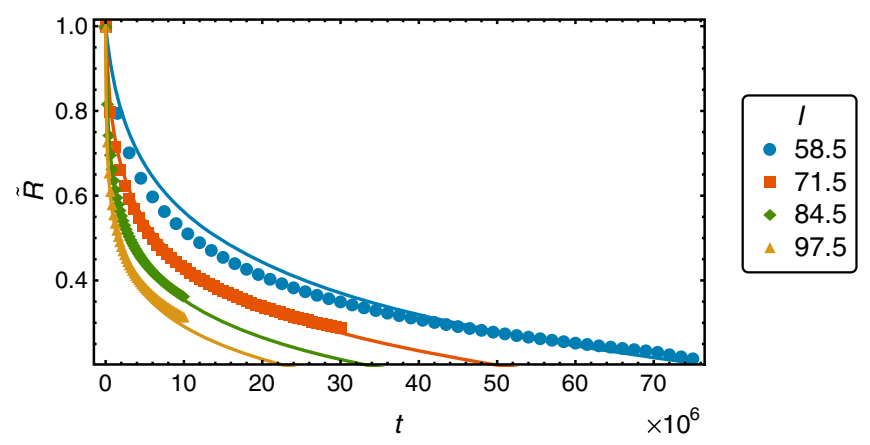

FIG. 9. Fitting results for the evolution of the normalized resistance according to Eq. (G2). $\delta R(t)=\frac{R_{\mathrm{HI}}-\frac{x_{\mathrm{int}}}{I} \ln \left(1+\frac{t}{\tau_{2}}\right)-R_{\mathrm{HI}}+\frac{x_{\mathrm{int}}}{I} \ln \left(1+\frac{\tau_{0}}{\tau_{2}}\right)}{R_{\mathrm{HI}}-R_{\mathrm{HI}}+\frac{x_{\mathrm{int}}}{I} \ln \left(1+\frac{\tau_{0}}{\tau_{2}}\right)}$,

and rescaling the time by $\tau_{0}$,

$$
\delta R\left(t / \tau_{0}\right)=1-\frac{\ln \left(1+\frac{\tau_{0}}{\tau_{2}} \frac{t}{\tau_{0}}\right)}{\ln \left(1+\frac{\tau_{0}}{\tau_{2}}\right)} .
$$

Notice that with the natural choice of simply setting $\tau_{0}$ as $\tau_{2}$, we obtain the scaling form presented in Eq. (8) of the main text.

However, in the experiment, we do not know a priori how to determine the characteristic time scale $\tau_{2}$, which is a strong function of the current $I$ and other material parameters. So, we adopt the following strategy. We use $\tau_{0}$ as a free scaling parameter, one for each $I$-dependent curve $R(t)$, which we rescale according to Eq. (G6). We vary the set of values $\tau_{0}[I]$ until we obtain a collapse of all the experimental and simulation curves. The results of the successful collapse are shown in Fig. 5 of the main text, where $R^{*}$ and $\Delta R$ in corresponding $y$ labels are defined through Eq. (G4) as $R^{*} \equiv R\left(\tau_{0}\right)$ and $\Delta R \equiv R_{\mathrm{HI}}-R\left(\tau_{0}\right)$, respectively. In our experience, the collapse is unique. We found a sole way to properly collapse the whole set of curves, which gave us further confidence on the adopted procedure. A crucial point now is that the collapsed set of curves could be fitted with the expression

$$
F(t)=1-\frac{\ln (1+c t)}{\ln (1+c)},
$$

with $c$ a current independent constant. We find the values $c=15.2$ for the experimental data and $c=29.4$ for the simulation ones. Hence, in regard to Eqs. (G6) and (G7), we observe that the constant $c$ is nothing but the ratio between the analytically established characteristic time $\tau_{2}$ and the empirically determined $\tau_{0}$, which are simply proportional to one another.

In Fig. 10, we plot the dependence of the scaling time as a function of the applied current $\tau_{0}(I)$. In the case of the simulation results, the data follow the same exponentially decreasing behavior as deduced for $\tau_{2}(I)$ [see Eq. (G3)]. Moreover, we find the ratio $\tau_{0}(I) / \tau_{2}(I)$ in good agreement with the constant $c=29.4$, which validates our practical scaling procedure for the determination of the characteristic switching time in the experimental case.

Under the same set of approximations that we have assumed in this section, plus the additional one of neglecting the effect of the background distribution of vacancies, one may also derive an explicit expression for the characteristic time $\tau_{1}$. We start from Eq. (E2), and similarly as before, adopt the approximation $\sinh \left(I R / x_{\text {int }}\right) \approx \frac{1}{2} \exp \left(I R / x_{\text {int }}\right)$. Then, making the substitution $y^{\prime}=1 / y$ in Eq. (E3) and assuming that the integral is 

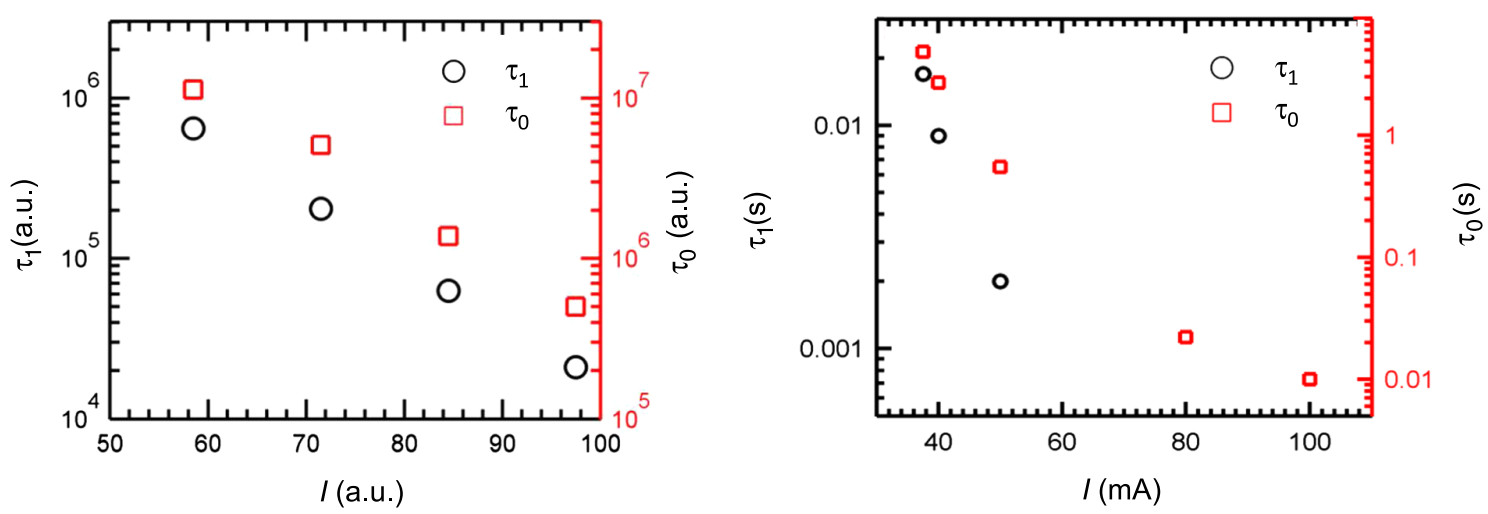

FIG. 10. Evolution of the characteristic times for different applied currents for both simulations (left panel) and experiments (right panel). It can be seen that both times follow an approximate exponential dependence with $I$ and that there exists a relative proportionality between them as predicted by our analysis.

dominated by the exponential factor (i.e., neglecting the change in lower-order factors), the integral has an analytical solution, and for the case with no background vacancies (i.e., $1 / \beta=0$ ), we get

$$
\tau_{1} \approx C\left(t_{0}, x_{0}\right)+\frac{x_{\mathrm{int}}^{2}}{D I R_{\mathrm{HI}}} \exp \left(-\frac{I R_{\mathrm{HI}}}{x_{\mathrm{int}}}\right),
$$

where the first term is the integration constant determined by the initial conditions $t_{0}$ and $x_{0}$. If this constant can be neglected (for example, for fast-forming shock waves), then we have the surprising result that $\tau_{1}=\tau_{2}$.

In the case of the model simulations, the results displayed in Fig. 10 show that, in fact, both characteristic times have the same $I$-dependent exponentially decaying behavior. Moreover, the ratio of the two characteristic times is approximately 25 , which is close to the constant $c=$ 29.4 quoted before; therefore, our simulations also validate the equality between the two characteristic times $\tau_{1}$ and $\tau_{2}$ predicted by the shock-wave scenario.

As may be expected, the experimental situation is only qualitatively consistent with the previous discussion. As seen in Fig. 10, for the experimentally determined characteristic times, we observe that they have an approximately similar dependence with the applied current; however, it is less clear if the equality between them also holds.

We show and compare the evolution of both characteristic times under different applied current in Fig. 10 for both simulations and experiments, observing a good agreement with the analysis presented here.

\section{APPENDIX H: QUANTITATIVE COMPARISON: EXPERIMENTS AND SIMULATIONS}

The magnitudes of the main parameters used in the simulations can be related to actual experimental magnitudes. In our model, all voltages are normalized by $k_{B} T / e$ and energies by $k_{B} T$. As experiments were performed at room temperature, we have $k_{B} T / e \approx 26 \mathrm{mV}$ and
$k_{B} T \approx 0.026 \mathrm{eV}$. In the simulations, the activation energy constant was given a value of $V_{0}=16 k_{B} T \approx 0.4 \mathrm{eV}$, which is consistent with experimental values reported for similar PCMO devices [42]. A correspondence can also be established for the voltages adopted in experiments and simulations. In the former, the applied voltages used for the resistive commutation were on the order of $1 \mathrm{~V}$. In units of $k_{B} T / e$, this leads to the dimensionless voltage values on the order of a few tens $(1 \mathrm{~V} / 26 \mathrm{mV}=38.5)$. However, in order to perform the extensive computational work required by our study within reasonable computational times, the values adopted in the simulations were higher by about 1 order of magnitude. For evident practical reasons (equipment and device limitations), such an increase of applied voltage cannot be performed in the experiments. Nevertheless, it should be clear from our theoretical discussion of the mathematical nature of the equations that the same qualitative results, namely, the formation of shock waves, also remain valid at lower applied voltages. Despite the significantly higher computational cost (days versus hours), we present here a set of runs for voltages that are close to the experimental values. In Fig. 11, we show the results for a simulation with an applied dimensionless current $I=6.75$, which corresponds to physical units $V=1.4 \mathrm{~V}$ since the initial (dimensionless) $R=8$ and then $V=I R k_{B} T / e=6.75 \times 8 \times 26 \mathrm{mV} \approx 1.4 \mathrm{~V}$. For convenience, in the figure, we also reproduce the corresponding results from the main text for a simulated voltage of $14.87 \mathrm{~V}$ ( $I=71.5$ a.u. $)$.

\section{APPENDIX I: SHOCK-WAVE FORMATION IN BINARY OXIDES}

In this section, we describe under what conditions one may expect the formation of shock waves to occur in binary insulating transition metal oxides such as $\mathrm{HfO}_{2}, \mathrm{TiO}_{2}, \mathrm{TaO}$, which are of current interest. These insulating systems are qualitatively different from the doped manganite compound that we consider in the main text. Nevertheless, it is 

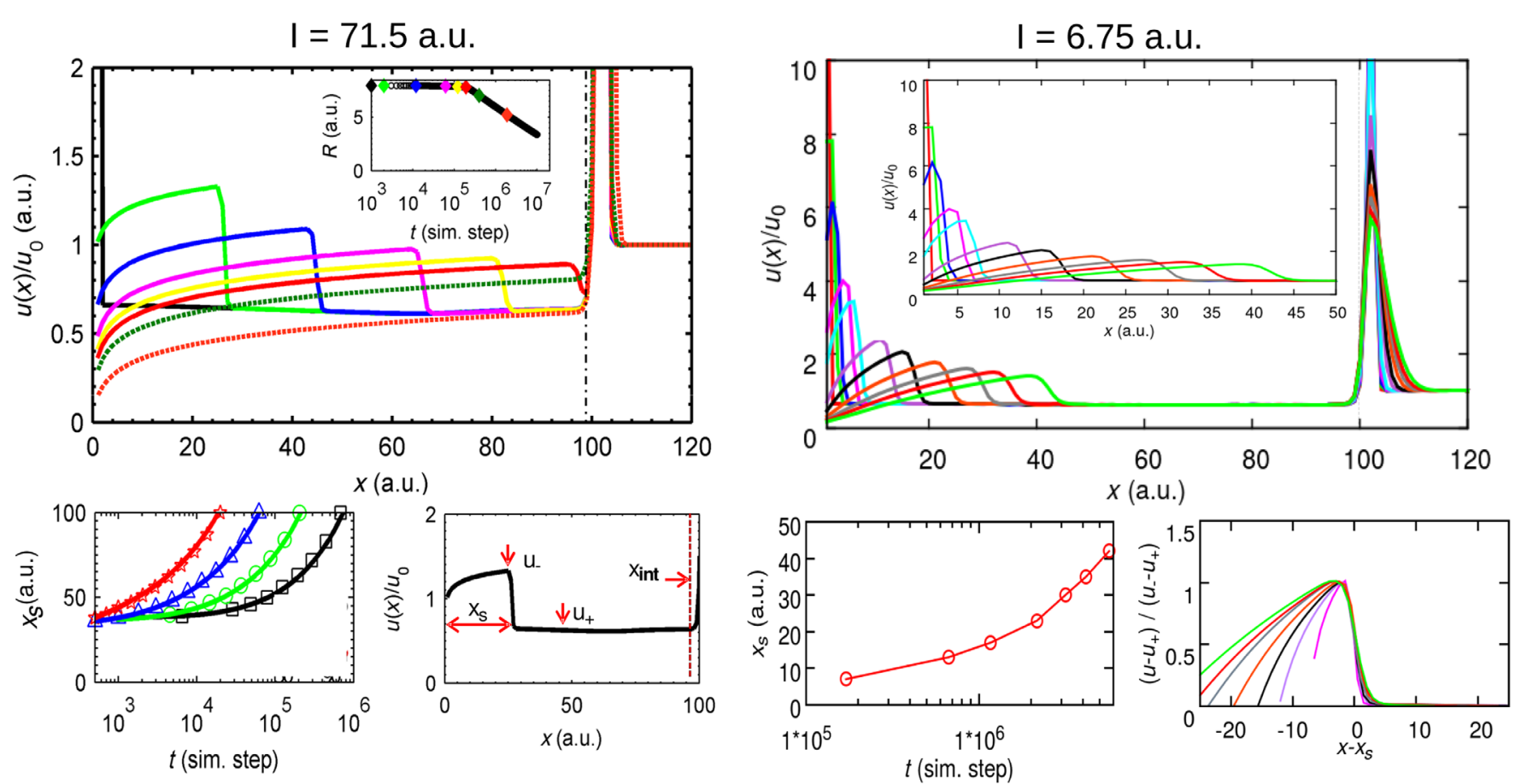

FIG. 11. Shock-wave dynamics for two different applied currents, which differ by nearly an order of magnitude. The lower-current case (right panel) corresponds to applied voltages comparable to the experimental ones, and the higher-voltage cases (left panel, same as Fig. 3) show similar shock-wave dynamics. The two lower-right panels demonstrate that (i) the propagation of the shock-wave front remains qualitatively the same as for higher applied currents, and (ii) the collapse of the snap shots of successive profiles indicates the formation of the shock wave.

interesting to realize that they may also sustain the formation of a shock-wave front according to our general formalism. However, a key point to realize is that the shock wave may occur for the propagation of the oxygen density profile and not the oxygen vacancy as in the manganite case. This is because in the latter, oxygen vacancies increase the local resistivity by means of creating defects in the oxygen-metal-oxygen bonds, hence in the conduction bands; in contrast, in the former case of binary systems, the oxygen vacancies dope electron carriers to an otherwise good insulator. Hence, the crucial features to realize that shock waves may occur in binary systems are the following: (i) The electroforming step renders the highly insulating system poorly conductive by creating a path with a massive production of oxygen vacancies. In an extreme case, this path may become a purely metallic filament, which leads to the so-called nonpolar resistive switching mode. However, if this is not so drastic, one gets to the bipolar switching mode with an intermediate density of oxygen vacancies. (ii) Within such a conductive path, oxygen ions move through the oxygen-vacancy sites by means of the applied electric field. As local oxygen density increases, the system locally approaches the stoichiometric formula, hence becoming locally more insulating with a local increase of resistivity. (iii) The local increase of resistivity leads to higher local voltage drops, and hence higher local electric fields, which further promote a higher motion of oxygen ions, leading to the formation of the shock wave. Interestingly, this phenomenon may have occurred in the simulation study of Strukov et al. [cf. Figs. 2(f) and 2(h) of Ref. [22]].

We now describe how the expressions of our shock-wave scenario derived for conductive manganites may be recast for insulating binary compounds. Let us consider $u_{\mathrm{O}}+u_{\mathrm{OV}}=n$, where $n$ is the integer for the oxygen atoms in the unit formula, and $u_{\mathrm{O}}$ and $u_{\mathrm{OV}}$ are the actual number of oxygen atoms and oxygen vacancies per unit formula in the sample, respectively; hence, they are proportional to the respective local densities. In the main text, we have derived the expressions in terms of $u$, the oxygen vacancies that corresponds to $u_{\mathrm{OV}}$. As we said before, we now focus on the oxygen density $u_{\mathrm{O}}$. Similar to the main text, we have a continuity equation for the oxygen, $\partial_{t} u_{\mathrm{O}}+\partial_{x} j_{\mathrm{O}}(t, x)=0$. From the components of drift and diffusion currents, we get

$$
\partial_{t} u_{\mathrm{O}}+f\left(u_{\mathrm{O}}\right) \partial_{x} u_{\mathrm{O}}=D \partial_{x x} u_{\mathrm{O}}
$$

where $f\left(u_{\mathrm{O}}\right) \equiv \partial_{u_{\mathrm{O}}} j_{\mathrm{O} \text {,drift }}\left(u_{\mathrm{O}}, I\right), \quad j_{\mathrm{O} \text {,diffusion }}=-D \partial_{x x} u_{\mathrm{O}}$, and $I(t)$ is the magnitude of the electronic current.

Notice that this equation is the generalized Burgers' equation for the case of oxygen-ion movement. The key point here is that, as pointed out in the main text, the function $f\left(u_{\mathrm{O}}\right)$ should be any monotonically increasing function of $u_{\mathrm{O}}$. Again, following the main text, 
$j_{\mathrm{O} \text {,drift }}=u_{\mathrm{O}} g(E)$, where $E$ is the magnitude of the local electric field and $g$ is clearly an increasing function of it. The specific form of $g(E)$ is material dependent, but the important point already mentioned is that a local increase of $u_{\mathrm{O}}$ leads to a local increase of $\rho\left(u_{\mathrm{O}}\right)$, and hence of $E$. Therefore, from analogous arguments as discussed in the main text, shock-wave fronts may also occur in the binaryinsulating compounds. As we said before, they may occur for systems in bipolar-mode RS. An interesting issue would be to investigate the temperature or current intensity dependence of the shock-wave formation. A priori, temperature may play two competing roles. On one hand, higher currents or fields would lead to higher temperatures, hence to higher oxygen-ion mobility. This may favor the formation of a shock wave. However, temperature also decreases the local resistivity of an insulator and hence may prevent the local increase of the field intensity.

Having discussed and established under which general conditions one may expect the formation of a shock wave, we now present the specific formulas for the behavior of the resistance $R(t)$ during the evolution of the shock-wave front. Figure 12 is a schematic representation of the time evolution of the shock wave and serves as the definition of the various relevant variables.

We assume that electroforming has created a path rich in oxygen vacancies, which dominates the conduction through the system. Thus, there is a constant background of density of oxygen $u_{\mathrm{O}}^{0}=u_{\mathrm{O},+}$ on top of which the shock wave evolves. The initial state is a high-resistive state with a pileup of oxygen atom (on the left-hand side) that brings the local density close to the stoichiometric value, and hence a highly insulating region. As a strong (positive) voltage is applied at the electrode at $L$, the oxygen ions migrate rapidly, forming a plateau of density $u_{\mathrm{O},-}$ with a sharp front of magnitude $u_{\mathrm{O},-}-u_{\mathrm{O},+}$ at the position $x_{s}$. The front moves with velocity $v_{s}$.

The total (two-point) resistance of the system (we neglect the effect of the electrode contacts) is given by

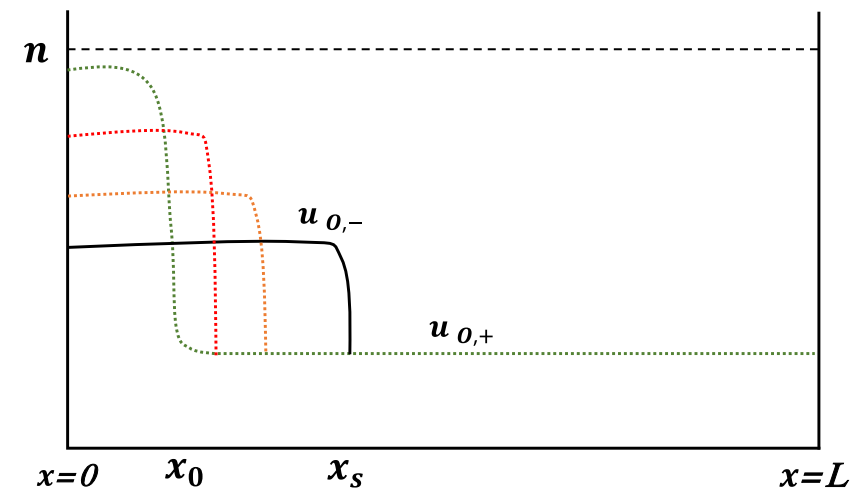

FIG. 12. The schematic plot for the oxygen shock-wave front propagation. The green curve is the initial distribution where oxygen is piled up at $x<x_{0}$.

$$
R(t)=\int_{x=0}^{x=L} \rho\left(u_{\mathrm{O}}(x)\right) d x .
$$

Using the continuity equation for oxygen, we can describe the decrease rate of the resistance from the high-resistive state $R_{\mathrm{HI}}$ to the low-resistive state $R_{\mathrm{LO}}$ as

$$
\begin{aligned}
\frac{d R(t)}{d t}= & -\int_{0}^{x_{s}}\left(\frac{d \rho}{d u_{\mathrm{O}}}\right) \partial_{x} j_{\mathrm{O}}\left(u_{\mathrm{O}}, t, x\right) d x \\
& -\int_{x_{s}}^{L}\left(\frac{d \rho}{d u_{\mathrm{O}}}\right) \partial_{x} j_{\mathrm{O}}\left(u_{\mathrm{O}}, t, x\right) d x \\
& +\rho\left(u_{\mathrm{O}}\left(x_{s}-\varepsilon\right)\right) v_{s}-\rho\left(u_{\mathrm{O}}\left(x_{s}+\varepsilon\right)\right) v_{s},
\end{aligned}
$$

where the quantity $\left(d \rho / d u_{\mathrm{O}}\right)$ is a function of $u_{\mathrm{O}}(t, x)$. The shock-wave velocity $v_{s}$ can be obtained via the RankineHugoniot condition as described in Appendix B. Since we assume a large initial pileup distribution of oxygen density near $x=0$, this creates a large local resistivity and thus large drift force; hence, we expect the shock wave will form quickly. Therefore, the distribution of $u_{\mathrm{O}}(t, x)$ for $x<x_{s}$ soon becomes approximately flat and will be denoted as $u_{O}\left(t, x_{s}-\varepsilon\right) \approx u_{\mathrm{O},-}(t)$.

Using the boundary condition $j_{\mathrm{O}}\left(u_{\mathrm{O}}, t, x=0\right)=$ $j_{\mathrm{O}}\left(u_{\mathrm{O}}, t, x=L\right)=0$, and defining $j_{\mathrm{O}}\left(u_{\mathrm{O}}, t, x_{s} \pm \varepsilon\right) \equiv$ $j_{\mathrm{O}, \pm}, \quad \rho_{ \pm} \equiv \rho\left(u_{\mathrm{O}}\left(x_{s} \pm \varepsilon\right)\right),\left.\quad \rho^{\prime}\left(u_{\mathrm{O}}\right)_{ \pm} \equiv\left(d \rho / d u_{\mathrm{O}}\right)\right|_{x_{s} \pm \varepsilon}$, $u_{\mathrm{O}}\left(x_{s} \pm \varepsilon\right) \equiv u_{\mathrm{O}, \pm}$, we have

$\frac{d R(t)}{d t}=-\left[\rho^{\prime}\left(u_{\mathrm{O}}\right)_{-} j_{\mathrm{O},-}-\rho^{\prime}\left(u_{\mathrm{O}}\right)_{+} j_{\mathrm{O},+}\right]+\left[\rho_{-}-\rho_{+}\right] v_{s}$.

Recalling that we assumed the background oxygen density to be approximately constant, we define $Q_{\mathrm{O}}$ as the total number of active oxygen ions, which is a constant during lapse of motion of the shock wave:

$$
Q_{\mathrm{O}}=\left[u_{\mathrm{O},-}(t)-u_{\mathrm{O},+}\right] x_{s}(t) .
$$

In terms of the parameters defined above, we have

$$
R(t)=\rho\left(u_{\mathrm{O},-}(t)\right) x_{s}(t)+\rho\left(u_{\mathrm{O},+}\right)\left(L-x_{s}(t)\right) .
$$

Then, taking the time derivative and comparing to Eq. (I4), we obtain

$$
\frac{d u_{\mathrm{O},-}}{d t}=\frac{j_{\mathrm{O},+} \rho^{\prime}\left(u_{\mathrm{O}}\right)_{+} / \rho^{\prime}\left(u_{\mathrm{O}}\right)_{-}-j_{\mathrm{O},-}}{x_{s}(t)},
$$

where $u_{\mathrm{O},+}$ is time independent and known, and similarly for $\rho^{\prime}\left(u_{\mathrm{O}}\right)_{+}, j_{\mathrm{O},+}$. To make further progress, we now need the specific forms of $\rho\left(u_{\mathrm{O}}\right)$ and $j_{\mathrm{O}}\left(u_{\mathrm{O}}, t, x\right)$, which depend on the specific transport properties of the physical system. Eliminating $x_{s}$, we could then obtain a differential equation for $u_{\mathrm{O},-}(t)$ : 
$\frac{d u_{\mathrm{O},-}}{d t}=\frac{\left(j_{\mathrm{O},+} \rho^{\prime}\left(u_{\mathrm{O}}\right)_{+} / \rho^{\prime}\left(u_{\mathrm{O}}\right)_{-}-j_{\mathrm{O},-}\right)\left(u_{\mathrm{O},-}-u_{\mathrm{O},+}\right)}{Q_{\mathrm{O}}}$,

and then, finally, we obtain the expression for $R(t)$ through Eq. (I6).

For a concrete illustration, we now assume a specific model for the conduction, where the local resistivity is an increasing function $u_{\mathrm{O}}$, as expected for the case of the binary insulators:

$$
\begin{aligned}
j_{\mathrm{O}} & =2 D u_{\mathrm{O}} \sinh \left[I \rho\left(u_{\mathrm{O}}\right)\right], \\
\rho\left(u_{\mathrm{O}}\right) & =\frac{\rho_{0}}{1+A_{0}\left(n-u_{\mathrm{O}}\right)} .
\end{aligned}
$$

This is motivated by the expected approximate linearity of the conductivity with the concentration of dopants, namely, oxygen vacancies, therefore we have, $\sigma=1 / \rho \sim u_{\mathrm{OV}}=$ $\left(n-u_{\mathrm{O}}\right)$. The parameter $A_{0}$ is a suitable constant, and $\rho_{0}$ is the intrinsic resistivity of the stoichiometric system (i.e., for $u_{\mathrm{OV}}=0$ ).

From the schematic in Fig. 12, we have $Q_{\mathrm{O}}=$ $\left[u_{\mathrm{O},-}(t=0)-u_{\mathrm{O},+}\right] x_{0}$, and we can solve Eq. (I8) using the explicit model defined in Eq. (I9). We may then solve (numerically) for the evolution of $u_{\mathrm{O},-}(t)$ and finally obtain the resistance as

$$
\begin{aligned}
R(t=0)= & R_{\mathrm{HI}}=\frac{\rho_{0} x_{0}}{1+A_{0}\left(n-u_{\mathrm{O},-}(t=0)\right)} \\
& +\frac{\rho_{0}\left(L-x_{0}\right)}{1+A_{0}\left(n-u_{\mathrm{O},+}\right)}, \\
R(t)= & \frac{\rho_{0} x_{s}}{1+A_{0}\left(n-u_{\mathrm{O},-}(t)\right)}+\frac{\rho_{0}\left(L-x_{s}\right)}{1+A_{0}\left(n-u_{\mathrm{O},+}\right)}, \\
R\left(t=t_{\text {final }}\right)= & R_{\mathrm{LO}}=\frac{\rho_{0} L}{1+A_{0}\left(n-u_{\mathrm{O},-}\left(t_{\text {final }}\right)\right)} .
\end{aligned}
$$

As a final remark, one may notice that from Eq. (I5) the resistance can be reparametrized as a function of the shockwave front position $x_{s}$,

$$
R\left[x_{s}(t)\right]=\frac{\rho_{0} x_{s}}{1+A_{0}\left(n-\frac{Q_{0}}{x_{s}}-u_{\mathrm{O},+}\right)}+\frac{\rho_{0}\left(L-x_{s}\right)}{1+A_{0}\left(n-u_{\mathrm{O},+}\right)},
$$

and $\quad R_{\mathrm{HI}}=R\left[x_{s}(t)=x_{0}\right], \quad R_{\mathrm{LO}}=R\left[x_{s}(t)=L\right] . \quad$ It $\quad$ is straightforward to see that $R\left[x_{s}(t)\right]$ is a monotonically decreasing function of $x_{s}(t)$ and thus $R_{\mathrm{LO}}<R_{\mathrm{HI}}$.

[1] International technology road map for semiconductors, http://www.itrs.net.
[2] M. J. Rozenberg, Resistive Switching, Scholarpedia 6, 11414 (2011).

[3] D. B. Strukov, G. S. Snider, D. R. Stewart, and R. S. Williams, The Missing Memristor Found, Nature (London) 453, 80 (2008).

[4] R. Waser and M. Aono, Nanoionics-Based Resistive Switching Memories, Nat. Mater. 6, 833 (2007).

[5] J. J. Yang, M. D. Pickett, X. Li, D. A. A. Ohlberg, D. R. Stewart, and R. S. Williams, Memristive Switching Mechanism for Metal/Oxide/Metal Nanodevices, Nat. Nanotechnol. 3, 429 (2008).

[6] R. Waser, R. Dittmann, G. Staikov, and K. Szot, RedoxBased Resistive Switching Memories-Nanoionic Mechanisms, Prospects, and Challenges, Adv. Mater. 21, 2632 (2009).

[7] I. H. Inoue and A. Sawa, Functional Metal Oxides, New Science and Novel Applications, edited by S. Ogale, T. Venkatesan, and M. Blamire (Wiley-VCH, Germany, 2013), Chap. 16.

[8] J. J. Yang, I. H. Inoue, T. Mikolajick, and C. S. Hwang, Metal Oxide Memories Based on Thermochemical and Valence Change Mechanisms, MRS Bull. 37, 131 (2012).

[9] A. Sawa, Resistive Switching in Transition Metal Oxides, Mater. Today 11, 28 (2008).

[10] A. Baikalov, Y. Q. Wang, B. Shen, B. Lorenz, S. Tsui, Y. Y. Sun, Y. Y. Xue, and C. W. Chu, Field-Driven Hysteretic and Reversible Resistive Switch at the Ag-Pr 0.7 Ca $0.3 \mathrm{MnO} 3$ Interface, Appl. Phys. Lett. 83, 957 (2003).

[11] P. Stoliar, P. Levy, M. J. Sánchez, A. G. Leyba, A. C. Albornoz, F. Gomez-Marlasca, A. Zanini, C. Toro Salazar, N. Ghenzi, and M. J. Rozenberg, Nonvolatile Multilevel Resistive Switching Memory Cell: A Transition Metal Oxide-Based Circuit, IEEE Trans. Circuits Syst. II: Express Briefs 61, 21 (2014).

[12] F. Alibart, L. Gao, B. D. Hoskins, and D. B. Strukov, High Precision Tuning of State for Memristive Devices by Adaptable Variation-Tolerant Algorithm, Nanotechnology 23, 075201 (2012).

[13] N. Ghenzi, M. J. Sánchez, F. Gomez-Marlasca, P. Levy, and M. J. Rozenberg, Hysteresis Switching Loops in Ag-Manganite Memristive Interfaces, J. Appl. Phys. 107, 093719 (2010).

[14] Y. B. Nian, J. Strozier, N. J. Wu, X. Chen, and A. Ignatiev, Evidence for an Oxygen Diffusion Model for the Electric Pulse Induced Resistance Change Effect in TransitionMetal Oxides, Phys. Rev. Lett. 98, 146403 (2007).

[15] X. Chen, N. J. Wu, J. Strozier, and A. Ignatiev, Direct Resistance Profile for an Electrical Pulse Induced Resistance Change Device, Appl. Phys. Lett. 87, 233506 (2005).

[16] S. Larentis, F. Nardi, S. Balatti, C. Gilme, and D. Ielmini, Resistive Switching by Voltage-Driven Ion Migration in Bipolar RRAM-Part II: Modeling, IEEE Trans. Electron Devices 58, 4309 (2011).

[17] S. Menzel, M. Waters, A. Marchewka, U. Bottger, R. Dittmann, and R. Waser, Origin of the Ultra-Nonlinear Switching Kinetics in Oxide-Based Resistive Switches, Adv. Funct. Mater. 21, 4487 (2011).

[18] M. Bocquet, D. Deleruyelle, H. Aziza, C. Muller, J. M. Portal, T. Cabout, and E. Jalaguier, Robust Compact Model 
for Bipolar Oxide-Based Resistive Switching Memories, IEEE Trans. Electron Devices 61, 674 (2014).

[19] J. H. Hur, M. J. Lee, C. B. Lee, Y. B. Kim, and C. J. Kim, Modeling for Bipolar Resistive Memory Switching in Transition-Metal Oxides, Phys. Rev. B 82, 155321 (2010).

[20] P. Huang, X. Y. Liu, B. Chen, H. T. Li, Y. J. Wang, Y. X. Deng, K. L. Wei, L. Zeng, B. Gao, G. Du, X. Zhang, and J. F. Kang, A Physics-Based Compact Model of Metal-OxideBased RRAM DC and AC Operations, IEEE Trans. Electron Devices 60, 4090 (2013).

[21] M. Noman, W. Jiang, P. A. Salvador, M. Skowronski, and J. A. Bain, Computational Investigations into the Operating Window for Memristive Devices Based on Homogeneous Ionic Motion, Appl. Phys. A 102, 877 (2011).

[22] D. B. Strukov, J. L. Borghetti, and R. S. Williams, Coupled Ionic and Electronic Transport Model of Thin-Film Semiconductor Memristive Behavior, Small 5, 1058 (2009).

[23] J. S. Lee, S. B. Lee, B. Kahng, and T. W. Noh, Two Opposite Hysteresis Curves in Semiconductors with Mobile Dopants, Appl. Phys. Lett. 102, 253503 (2013).

[24] S. Kim, S. Choi, and W. Lu, Comprehensive Physical Model of Dynamic Resistive Switching in an Oxide Memristor, ACS Nano 8, 2369 (2014).

[25] M.J Rozenberg, I. H. Inoue, and M. J. Sanchez, Nonvolatile Memory with Multilevel Switching: A Basic Model, Phys. Rev. Lett. 92, 178302 (2004).

[26] U. Russo, D. Ielmini, C. Cagli, and A. L. Lacaita, Filament Conduction and Reset Mechanism in NiO-Based ResistiveSwitching Memory (RRAM) Devices, IEEE Trans. Electron Devices 56, 186 (2009).

[27] M. J. Rozenberg, M. J. Sanchez, R. Weht, C. Acha, F. Gomez-Marlasca, and P. Levy, Mechanism for Bipolar Resistive Switching in Transition-Metal Oxides, Phys. Rev. B 81, 115101 (2010).

[28] L. Debnath, Nonlinear Partial Differential Equations for Scientists and Engineers (Birkhauser, Boston, 2011).

[29] M. Taylor, Partial Differential Equations I, II, III, Applied Mathematical Sciences (Springer, New York, 2011).

[30] While the drift current contains the nonlinear term that leads to a steep wave front, according to Fick's law, the diffusion current flows in such a way as to reduce the steepness of the density profile. Therefore, the diffusion current stabilizes the propagation of the shock wave and protects the solution from entering the self-breaking region (multivalued solution). In the scenario of the standard Burgers' equation, where $f(u) \sim u$ in Eq. (1), the equation can be explicitly solved via Cole-Hopf transformation (see Refs. [28,29]). In that case, we can check the effects of the diffusion current against nonlinear drift exactly.
[31] R. Courant and D. Hilbert, Methods of Mathematical Physics. Vol. II: Partial Differential Equations (Interscience, New York, 1962).

[32] H.-S. Lee, S.-G. Choi, H.-H. Park, and M. J. Rozenberg, A New Route to the Mott-Hubbard Metal-Insulator Transition: Strong Correlations Effects in $\operatorname{Pr}_{0.7} \mathrm{Ca}_{0.3} \mathrm{MnO}_{3}$, Sci. Rep. 3, 17 (2013).

[33] L. D. Landau and E. M. Lifshitz, Fluid Mechanics: Volume 6 (Course of Theoretical Physics) (Butterworth-Heinemann, Oxford, 1987).

[34] M. Quintero, P. Levy, A. G. Leyva, and M. J. Rozenberg, Mechanism of Electric-Pulse-Induced Resistance Switching in Manganites, Phys. Rev. Lett. 98, 116601 (2007).

[35] Y. 1. Jin, Z.t. Xu, K. J. Jin, X. He, C. Wang, and H. b. Lu, Enhancement of Resistive Switching Effect in Double-Layered Pt/Pr0.7Ca0.3MnO3/LaO.6Pr0.4MnO3/ SrNb0.01Ti0.9903 Heterostructure, Physica B (Amsterdam) 449, 52 (2014).

[36] M. Scherff, J. Hoffmann, B. Meyer, T. Danz, and C. Jooss, Interplay of Cross-Plane Polaronic Transport and Resistive Switching in Pt-Pr0.67Ca0.33MnO3-Pt Heterostructures, New J. Phys. 15, 103008 (2013).

[37] S. Park, S. Jung, M. Siddik, M. Jo, J. Park, S. Kim, W. Lee, J. Shin, D. Lee, G. Choi, J. Woo, and E. Cha, Self-Formed Schottky Barrier Induced Selector-Less RRAM for CrossPoint Memory Applications, Physica Status Solidi-Rapid Research Lett. 6, 454 (2012).

[38] A. Herpers, C. Lenser, C. Park, F. Offi, F. Borgatti, G. Panaccione, S. Menzel, R. Waser, and R. Dittmann, Spectroscopic Proof of the Correlation between RedoxState and Charge-Carrier Transport at the Interface of Resistively Switching Ti/PCMO Devices, Adv. Mater. 26, 2730 (2014).

[39] F. Gomez-Marlasca, N. Ghenzi, A. G. Leyba, A. C. Albornoz, D. Rubi, P. Stoliar, and P. Levy, Modeling Electronic Transport Mechanisms in Metal-Manganite Memristive Interfaces, J. Appl. Phys. 113, 144510 (2013).

[40] N. Ghenzi, M. J. Rozenberg, R. Llopis, P. Levy, L. E. Hueso, D. Rubi, and P. Stoliar, Tuning the Resistive Switching Properties of TiO2-x Films, Appl. Phys. Lett. 106, 123509 (2015).

[41] It is straightforward to see that $u$ is constant along the characteristics since $d u / d t=d x / d t \partial_{x} u+\partial_{t} u=0$.

[42] Y. B. Nian, J. Strozier, N. J. Wu, X. Chen, and A. Ignatiev, Evidence for an Oxygen Diffusion Model for the Electric Pulse Induced Resistance Change Effect in TransitionMetal Oxides, Phys. Rev. Lett. 98, 146403 (2007).

[43] There is, in fact, a small migration of background vacancies into the bulk during the period of shock-wave propagation which causes a small variation of the resistance during this phase. 